\title{
Unforced Surface Air Temperature Variability and Its Contrasting Relationship with the Anomalous TOA Energy Flux at Local and Global Spatial Scales*
}

\author{
PATRICK T. BROWN AND WENHONG LI \\ Earth and Ocean Sciences, Nicholas School of the Environment, Duke University, Durham, North Carolina \\ JONATHAN H. JIANG AND HUI SU \\ Jet Propulsion Laboratory, California Institute of Technology, Pasadena, California
}

(Manuscript received 29 May 2015, in final form 6 November 2015)

\begin{abstract}
Unforced global mean surface air temperature $(\bar{T})$ is stable in the long term primarily because warm $\bar{T}$ anomalies are associated with enhanced outgoing longwave radiation ( $\uparrow \mathrm{LW})$ to space and thus a negative net radiative energy flux ( $\bar{N}$, positive downward) at the top of the atmosphere (TOA). However, it is shown here that, with the exception of high latitudinal and specific continental regions, warm unforced surface air temperature anomalies at the local spatial scale $[T(\theta, \phi)$, where $(\theta, \phi)=$ (latitude, longitude)] tend to be associated with anomalously positive $N(\theta, \phi)$. It is revealed that this occurs mainly because warm $T(\theta, \phi)$ anomalies are accompanied by anomalously low surface albedo near sea ice margins and over high altitudes, low cloud albedo over much of the middle and low latitudes, and a large water vapor greenhouse effect over the deep Indo-Pacific.

It is shown here that the negative $\bar{N}$ versus $\bar{T}$ relationship arises because warm $\bar{T}$ anomalies are associated with large divergence of atmospheric energy transport over the tropical Pacific [where the $N(\theta, \phi)$ versus $T(\theta, \phi)$ relationship tends to be positive] and convergence of atmospheric energy transport at high latitudes [where the $N(\theta, \phi)$ versus $T(\theta, \phi)$ relationship tends to be negative]. Additionally, the characteristic surface temperature pattern contains anomalously cool regions where a positive local $N(\theta, \phi)$ versus $T(\theta, \phi)$ relationship helps induce negative $\bar{N}$. Finally, large-scale atmospheric circulation changes play a critical role in the production of the negative $\bar{N}$ versus $\bar{T}$ relationship as they drive cloud reduction and atmospheric drying over large portions of the tropics and subtropics, which allows for greatly enhanced $\uparrow \mathrm{LW}$.
\end{abstract}

\section{Introduction}

Changes in surface air temperature $(T)$ can be caused by external radiative forcings that impose a net radiative energy flux ( $N$, positive down) at the top of the atmosphere (TOA):

$$
N=\downarrow \mathrm{SW}-\uparrow \mathrm{SW}-\uparrow \mathrm{LW},
$$

where SW represents shortwave (solar) radiation at the TOA, LW represents longwave (terrestrial) radiation at

\footnotetext{
* Supplemental information related to this paper is available at the Journals Online website: http://dx.doi.org/10.1175/JCLI-D-150384.s1.

Corresponding author address: Patrick T. Brown, Earth and Ocean Sciences, Nicholas School of the Environment, Duke University, 5120K Environment Hall, 9 Circuit Drive, Durham, NC 27708.

E-mail: patrick.brown@duke.edu
}

the TOA, and the arrows represent the direction of the flux. Additionally, $T$ experiences unforced variability that originates from the internal dynamics of the climate system (Brown et al. 2014a; Hasselmann 1976; Hawkins and Sutton 2009; Palmer and McNeall 2014). Unforced variability in global mean $T(\bar{T})$ has generated great scientific and public interest as it has the ability to either enhance or obscure externally forced signals such as the long-term warming due to increased greenhouse gas concentrations (Brown et al. 2015). Much work on the causes of unforced $\bar{T}$ variability has focused on changes in the net heat flux between the ocean and atmosphere (Chen and Tung 2014; Drijfhout et al. 2014; England et al. 2014; Meehl et al. 2013). However, it is also recognized that unforced variability in $N$, due to changes in clouds in particular, can enhance the magnitude and persistence of unforced $T$ variability locally (Bellomo et al. 2014, 2015; Evan et al. 2013; Trzaska et al. 2007). Therefore, there has been substantial interest in the 


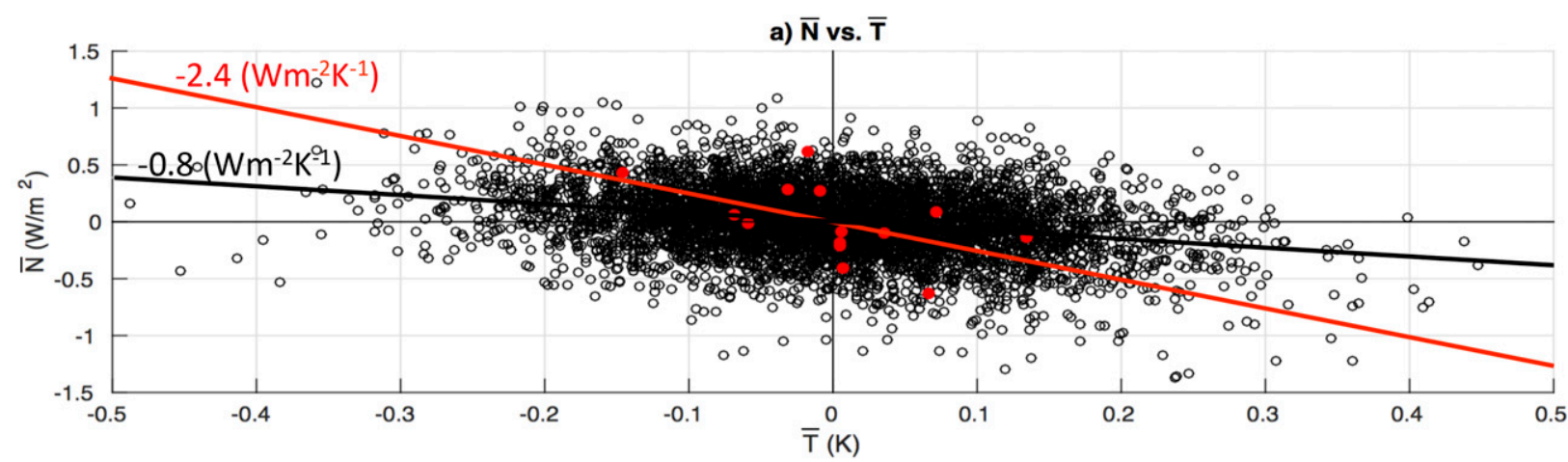

b) AOGCMs: $\mathbf{N}(\alpha, \phi)$ vs. $\mathbf{T}(\alpha, \phi),(\mathbf{A V G}=1.9)$

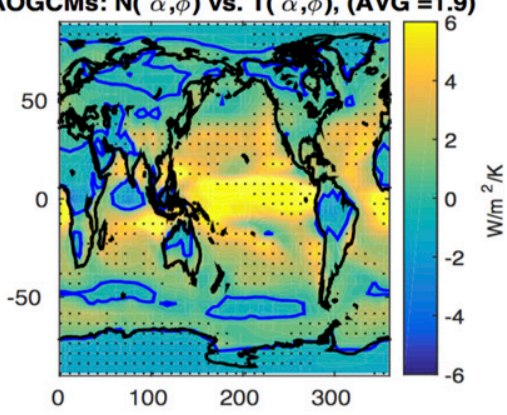

c) Obs: $\mathbf{N}(\alpha, \phi)$ vs. $\mathbf{T}(\alpha, \phi),(\mathrm{AVG}=1.4)$

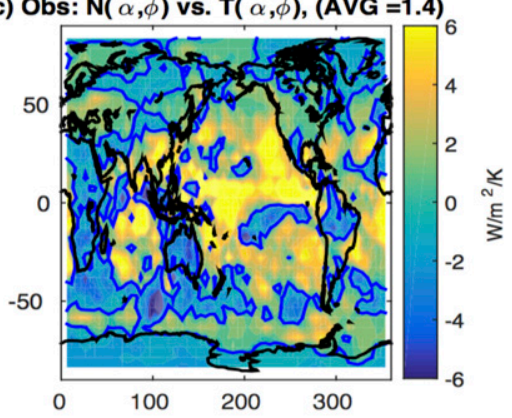

d) $\mathbf{N}(\alpha, \phi)$ vs. $\mathbf{T}(\alpha, \phi)$, Zonal Mean

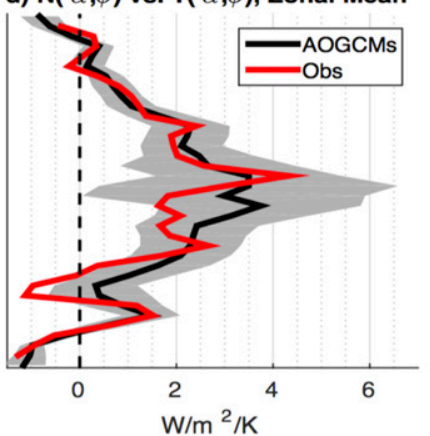

FIG. 1. Sign difference between the global mean $\bar{N}$ vs $\bar{T}$ and the local $N(\theta, \phi)$ vs $T(\theta, \phi)$ relationship. (a) Linear least squares relationship between annual mean $\bar{N}$ and annual mean $\bar{T}$ pooled across 27 CMIP5 AOGCMs (black), and in CERES/ERA-I observations from 2001-14 (red). (b) Map of the multimodel means of the linear least squares regression coefficients between annual anomalous $N(\theta, \phi)$ and annual anomalous $T(\theta, \phi)$. The zero isopleth is contoured in blue and stippling represents where at least $90 \%$ of the models agree on the sign of the regression coefficient. (c) As in (b), but for CERES/ERA-I observations from 2001-14. (d) Zonal mean of (b) and (c) with gray shading representing the across-AOGCM standard deviation of the zonal mean of the regression values.

relationship between $\bar{T}$ and $\bar{N}$ in the context of unperturbed climate variability (Allan et al. 2014; Brown et al. 2014a; Kato 2009; Loeb et al. 2012; Palmer and McNeall 2014; Smith et al. 2015; Trenberth et al. 2014) as this relationship may have implications for the magnitude and persistence of unforced $\bar{T}$ variability.

When the climate system is unperturbed by external radiative forcings, it is expected that $\bar{T}$ would be stable on long time scales predominantly because of the Planck response, or the direct blackbody radiative response to a uniform temperature change of the surface and the atmosphere (Bony et al. 2006; Dessler 2013; Hallberg and Inamdar 1993; Ingram 2013). In the global mean sense, the Planck response suggests that positive $\bar{T}$ anomalies tend to be associated with enhanced $\uparrow \mathrm{LW}$, which would cause negative $\bar{N}$, and thus an eventual return of $\bar{T}$ to its equilibrium value (Brown et al. 2014a; Dessler 2013; Koumoutsaris 2013; Trenberth et al. 2015). Indeed, both satellite observations and atmosphere-ocean general circulation models (AOGCMs) show this negative $\bar{N}$ versus $\bar{T}$ global mean relationship for interannual variability (Fig. 1a).

It may be tempting to suppose that this negative $\bar{N}$ versus $\bar{T}$ global relationship should hold at the local spatial scale as well, but it was recently pointed out that the $N(\theta, \phi)$ versus $T(\theta, \phi)$ relationship [where $(\theta, \phi)=$ (latitude, longitude)] is in fact positive in observations over much of Earth (Trenberth et al. 2015). Indeed, the same observations and AOGCMs that demonstrate the negative $\bar{N}$ versus $\bar{T}$ relationship (Fig. 1a) indicate that the $N(\theta, \phi)$ versus $T(\theta, \phi)$ relationship tends to be positive over most of the surface of the planet (Figs. 1b-d). The underlying reasons for the spatial distribution of the $N(\theta, \phi)$ versus $T(\theta, \phi)$ relationship as well as the cause of the relationship's sign reversal at the global scale are the primary topics of investigation in this study. Elucidating these relationships will improve our physical understanding of unforced $T$ variability and may improve efforts to model climate variability on both local and global scales.

\section{Data, preprocessing, and definitions}

\section{a. AOGCM data}

We focus on the relationship between unforced anomalous annual mean $T$ and unforced anomalous 
$\bar{T}$ and $\bar{N}$ for each AOGCM in this study

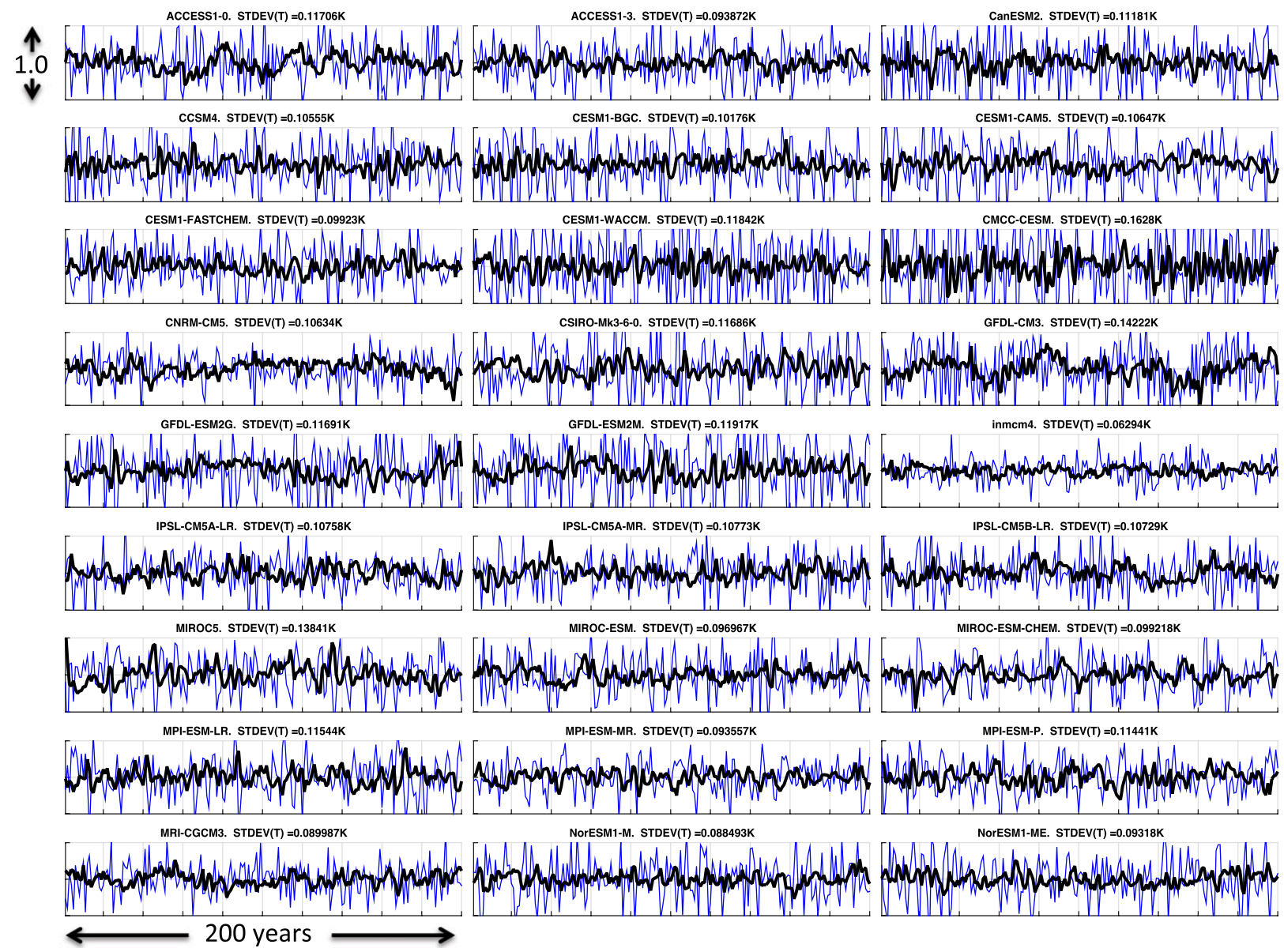

FIG. 2. Global mean temperature $(\mathrm{K})$ and net TOA radiation $\left(\mathrm{W} \mathrm{m}^{-2}\right)$ for the 27 AOGCM preindustrial control runs used in this this study. Time series of $\bar{T}$ are black and time series of $\bar{N}$ are blue.

annual mean energy fluxes in 27 AOGCMs that participated in phase 5 of the Coupled Model Intercomparison Project (CMIP5; Taylor et al. 2012). Details on the AOGCMs used in this study can be found in Table S1 in the supplementary material. We utilized unforced preindustrial control runs, which included no external radiative forcings, and thus all variability emerged spontaneously from the internal dynamics of the modeled climate system. We used the first 200 years of each AOGCM's preindustrial control run and linearly detrended all analyzed variables so that our analysis was not contaminated by possibly unphysical model drift (Fig. 2).

We focus our analysis on multimodel mean values (e.g., the mean of the AOGCMs' $N$ vs $T$ linear regression coefficients) in order to highlight the most robust relationships across the ensemble. However, the AOGCM spread about these mean values is shown where appropriate (e.g., Figs. 1d and 10; see also Fig. S6 in the supplementary material) and we indicate model agreement with stippling that denotes where more than $90 \%$ of AOGCMs agree on the sign of the regression coefficients (e.g., Figs. 1b, 3a-k, and 6a-k).

\section{b. Observational data}

We supplement the AOGCM analysis with TOA radiation measurements from the Clouds and Earth's Radiant Energy System (CERES; Wielicki et al. 1996) Energy Balanced and Filled (EBAF, version 2.8) product and cloud area fraction from the CERESMODIS product (Minnis et al. 2011). Additionally, we use the European Center for Medium-Range Weather Forecasts interim reanalysis (ERA-Interim, hereinafter ERA-I; Dee et al. 2011) to provide historical estimates of $T$, sea level pressure (SLP), and surface heat flux $(S)$. We use annual mean values for these datasets over the 14-yr period in which they overlap (2001-14). For simplicity we refer to both ERA-I and CERES data as 
observations even though ERA-I output represents observations assimilated into a weather forecast model. We linearly detrend all of the observations prior to further analysis. It should be noted that the historical record contains a combination of both forced and unforced variability; these are difficult to disentangle but over the relatively short time period of investigation (2001-14) unforced variability accounts for a substantial majority of the observed variation (Dessler 2010; Trenberth et al. 2010).

The purpose of this manuscript is to use both AOGCMs and observations to gain physical insight on the covariability between $N$ and $T$. Therefore, it is not our intent to rigorously compare AOGCMs to observations in order to assess model performance. Nevertheless, AOGCMs are known to struggle with the simulation of clouds, and thus it is useful to keep in mind that there are some large differences between AOGCM-modeled and observed cloud climatologies (Fig. S1 in the supplementary material). See also Dolinar et al. (2015) for further discussion.

\section{c. Definitions}

\section{1) LocAl AND GLOBAL SPATIAL SCALE}

We bilinearly interpolate all variables $(\alpha)$ from both AOGCM and observational datasets to a common $2.5^{\circ} \times 2.5^{\circ}$ latitude-longitude $[(\theta, \phi)]$ grid. Global mean values, denoted by an overbar, are calculated as

$$
\bar{\alpha}=\frac{1}{A} \sum_{i}^{M} a_{i}[\alpha(\theta, \phi)]_{i},
$$

where the $i$ subscript indicates the $i$ th value of a total of $M$, which is weighed by the grid box area $a_{i}$ and normalized by the total Earth surface area $A$.

\section{2) Components of $N$}

To gain insight into the underlying physics governing $N$ variability, we follow previous studies (Ramanathan et al. 1989) to decompose $N$ into four linearly additive components:

$$
\begin{aligned}
& \text { Clear }_{\text {SW }}=\left[\downarrow S W-\uparrow S W_{\text {clear_sky }}\right], \\
& \text { Clear }_{\text {LW }}=\left[-\uparrow \mathrm{LW}_{\text {clear_sky }}\right] \text {, } \\
& \mathrm{CRE}_{\mathrm{SW}}=\left[\downarrow \mathrm{SW}-\uparrow \mathrm{SW}_{\text {all_sky }}\right]-\left[\downarrow \mathrm{SW}-\uparrow \mathrm{SW}_{\text {clear_sky }}\right] \\
& =\uparrow \mathrm{SW}_{\text {clear_sky }}-\uparrow \mathrm{SW}_{\text {all_sky }} \text {, and } \\
& \mathrm{CRE}_{\mathrm{LW}}=\left[-\uparrow \mathrm{LW}_{\text {all_sky }}\right]-\left[-\uparrow \mathrm{LW}_{\text {clear_sky }}\right] \\
& =\uparrow \mathrm{LW}_{\text {clear_sky }}-\uparrow \mathrm{LW}_{\text {all_sky }},
\end{aligned}
$$

where Clear $_{\mathrm{Sw}}, \mathrm{Clear}_{\mathrm{Lw}}, \mathrm{CRE}_{\mathrm{Sw}}$, and $\mathrm{CRE}_{\mathrm{Lw}}$ represent the anomalous clear-sky shortwave, anomalous clear-sky longwave, anomalous cloud radiative effect (CRE) shortwave, and CRE longwave components, respectively, at the TOA (all positive downward). We also investigate the net impact of clouds using

$$
\mathrm{CRE}=\mathrm{CRE}_{\mathrm{SW}}+\mathrm{CRE}_{\mathrm{LW}} .
$$

The CRE is a measure of the impact of cloud radiative properties and cloud fraction on the TOA radiation budget relative to a cloudless atmosphere (Ramanathan et al. 1989). Thus, a change in the CRE with $T$ is not a pure measure of cloud feedback since a change in the CRE can occur because of a change in clouds or a change in the clear-sky radiation budget (Soden et al. 2004). This makes it difficult to isolate the effect of clouds on $N$ over regions with large changes in the clearsky energy budget. Nevertheless, decomposing $N$ using Eqs. (3)-(7) provides some physical insight that would not be available otherwise. In future work it may be valuable to investigate the components of $N$ using different methods such as the partial radiative perturbation technique (Donohoe and Battisti 2011).

\section{3) SuRfACE AND ATMOSPHERIC ENERGY FLUXES}

The net anomalous upward surface heat flux $(S)$ is

$$
S=\uparrow \mathrm{LE}+\uparrow \mathrm{SH}+\left[\uparrow \mathrm{SW}_{S}-\downarrow \mathrm{SW}_{S}\right]+\left[\uparrow \mathrm{LW}_{S}-\downarrow \mathrm{LW}_{S}\right],
$$

where LE is the anomalous latent heat flux, $\mathrm{SH}$ is the anomalous sensible heat flux, $\mathrm{SW}_{S}$ is the anomalous shortwave radiation flux, and $\mathrm{LW}_{S}$ is the anomalous longwave radiation flux all defined at Earth's surface under all-sky conditions.

We follow (Trenberth et al. 2002a,b) to define an estimate of the convergence of the vertically integrated atmospheric energy transport (AET):

$$
-\nabla \cdot \operatorname{AET}(\theta, \phi)=-1[N(\theta, \phi)+S(\theta, \phi)] .
$$

For observations, both $S(\theta, \phi)$ and $N(\theta, \phi)$ in Eq. (9) came from ERA-I [rather than using $N(\theta, \phi)$ from CERES] so that potentially disparate datasets were not mixed. This approximation ignores any atmospheric storage of heat, which was assumed to be small.

\section{4) LINEAR REGRESSION RELATIONSHIPS}

In the sections below we will make use of the following notation to denote a variety of different linear least squares regression relationships between climatic variables $(\alpha)$ and $T$ both on the local $[T(\theta, \phi)]$ and global $[\bar{T}]$ scales. 
The regression coefficient between any global mean variable $\bar{\alpha}$ and $\bar{T}$ is denoted as

$$
\overline{\gamma_{\alpha}}=\frac{\Delta \bar{\alpha}}{\Delta \bar{T}}
$$

The corresponding regression coefficient at the local spatial scale is denoted as

$$
\gamma_{\alpha}(\theta, \phi)=\frac{\Delta[\alpha(\theta, \phi)]}{\Delta[T(\theta, \phi)]} .
$$

Note that the global mean of the regression coefficients calculated on the local scale $\left[\overline{\gamma_{\alpha}(\theta, \phi)}\right]$ is not the same quantity as the regression coefficient calculated on global means $\left[\overline{\gamma_{\alpha}}\right]$ as Fig. 1 demonstrates.

Finally, the linear relationship between a variable defined at the local spatial scale and $\bar{T}$ is denoted as

$$
\zeta_{\alpha}(\theta, \phi)=\frac{\Delta[\alpha(\theta, \phi)]}{\Delta \bar{T}} .
$$

\section{5) FeEdBACKS}

We follow convention by referring to the linear relationship between a TOA radiative flux anomaly and a $T$ anomaly as a "feedback" (Bellomo et al. 2015; Colman and Power 2010; Dessler 2013; Koumoutsaris 2013; Trenberth et al. 2015). This language can give the impression that we know the change in $T$ is the cause and the change in TOA flux is the effect. It is safe to assume this direction of causality when an external forcing is obviously responsible for the $T$ change but the direction of causality is more ambiguous in the unforced climate state where all variability is spontaneously generated by the system itself. Undoubtedly there are instances where changes in the TOA flux (e.g., atmospheric circulation induced changes in clouds over land) lead to the $T$ anomaly (Trenberth and Shea 2005). Therefore, we caution that we use the term feedback to be consistent with other contemporary work on this subject but we do not wish to convey that the direction of causality is necessarily known in all cases.

\section{The geographic distribution of the $\gamma_{N}(\theta, \phi)$ relationship}

We first investigate the local relationships between $N$ and $T\left[\gamma_{N}(\theta, \phi)\right.$; Eq. (11)] with the intent of uncovering the physical processes underlying these relationships as well as how these physical processes differ by geographic location. Figure 3 maps $\gamma_{\alpha}(\theta, \phi)$ for a number of variables in both AOGCMs (Figs. 3a-k) and observations (Fig. 31-v). Note that $\gamma_{\text {Clear LW }}(\theta, \phi)$ (Figs. 3e,p) is affected by the lapse rate feedback, the water vapor feedback, and the Planck response (Colman and Power 2010; Crook et al. 2011), but over most of Earth's surface the Planck response dominates this component and there is enhanced $\uparrow \mathrm{LW}(\theta, \phi)$ to space during elevated $T(\theta, \phi)$. [Note that $\uparrow \mathrm{LW}$ via the Planck response is more heavily influenced by tropospheric mean temperature than by $T$ itself but that $T$ and tropospheric mean temperature are positively correlated on these time scales (Trenberth et al. 2015).] In the AOGCMs, the primary exception to this enhanced $\uparrow \mathrm{LW}(\theta, \phi)$ with warm $T(\theta, \phi)$ is over the Indo-Pacific warm pool where higher climatological surface temperatures allow for a water vapor response that is strong enough to overwhelm the Planck response (Allan et al. 1999; Inamdar and Ramanathan 1994; Larson and Hartmann 2003; Pierrehumbert 1995; Ramanathan and Collins 1991; Su et al. 2006). In this region, anomalous warmth is also associated with enhanced convection and cloud fraction (Fig. 3k) but since the shortwave (Fig. 3c) and longwave (Fig. 3f) CRE components mostly cancel (Fig. 3i) (Kiehl 1994), it is the water vapor feedback (Fig. 3e) that is primarily responsible for the positive $\gamma_{N}(\theta, \phi)$ relationship there. Note that the strength of the water vapor response also depends on enhanced convection as moistening of the middle and upper troposphere is crucial for its large magnitude in this region (Hallberg and Inamdar 1993).

Observations tell a similar story except that $\gamma_{\text {Clear LW }}(\theta, \phi)$ is negative over the central portion of the Indo-Pacific warm pool (Fig. 3p). This disagreement may be because satellites are only able to sample $\operatorname{Clear}_{\mathrm{LW}}(\theta, \phi)$ in regions that are actually cloud-free [unlike AOGCMs which calculate $\operatorname{Clear}_{\mathrm{LW}}(\theta, \phi)$ at all grid points and at every time step regardless of the simulated cloud cover]. A consequence of this is that the $\operatorname{Clear}_{\mathrm{LW}}(\theta, \phi)$ measurement from satellites will disproportionately represent the cloudless areas with less humidity and less of a water vapor greenhouse effect.

Over most of the remainder of the surface, with the exception of the subpolar latitudes, the positive $\gamma_{N}(\theta, \phi)$ relationship is due mostly to the $\gamma_{\mathrm{CRESW}}(\theta, \phi)$ component (Figs. 3c,n) associated with a reduction in cloud fraction (Figs. $3 \mathrm{k}, \mathrm{v}$ ) and an overall positive $\gamma_{\mathrm{CRE}}(\theta, \phi)$ (Figs. 3i,t). This is consistent with the shortwave cloud feedback that has been noted in regions characterized by high-albedo, low-level stratiform clouds in particular (Evan et al. 2013; Park et al. 2005). In these regions, elevated $T(\theta, \phi)$ is associated with increased convection and destabilization of the boundary layer (Bellenger et al. 2014) as well as a lack of sufficient increase in evaporation to maintain the boundary layer cloudiness 
$\gamma_{\alpha}(\theta, \varnothing)=\frac{\Delta[\alpha(\theta, \varnothing)]}{\Delta[T(\theta, \varnothing)]}($ where $\alpha=$ any variable $)$

(i.e. what happens when local T is high...)

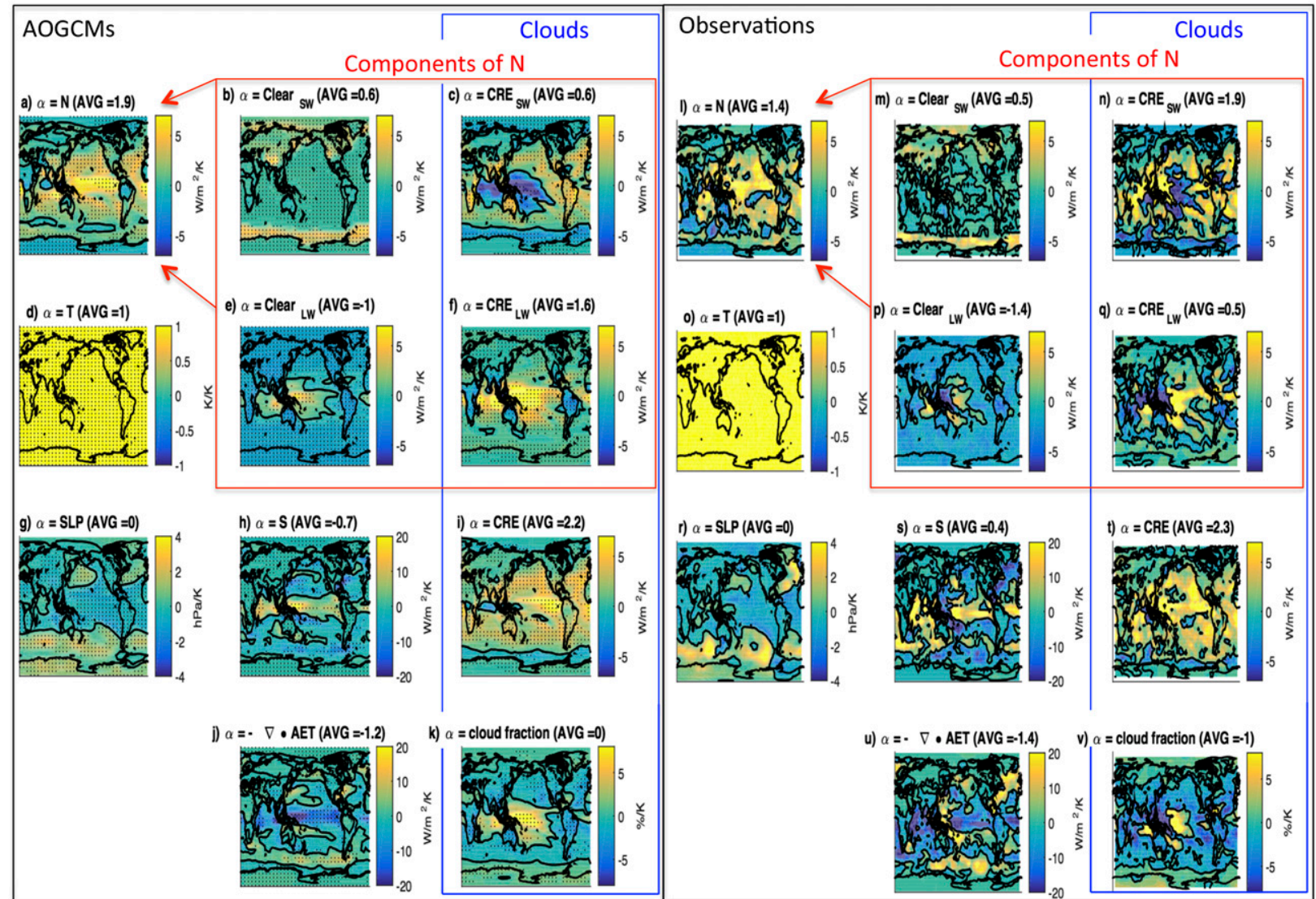

FIG. 3. Maps of variables defined locally, $\alpha(\theta, \phi)$ regressed against anomalous local temperature, $T(\theta, \phi)$ [Eq. (11)]. Multimodel mean of the annual linear least squares regression coefficients of (a) $N(\theta, \phi)$, (b) $\operatorname{Clear}_{\mathrm{Sw}}(\theta, \phi)$, (c) $\mathrm{CRE}_{\mathrm{Sw}}(\theta, \phi),(\mathrm{d}) T(\theta, \phi),(\mathrm{e}) \mathrm{Clear} \mathrm{LW}_{\mathrm{L}}(\theta, \phi)$, (f) $\operatorname{CRE}_{\mathrm{Lw}}(\theta, \phi),(\mathrm{g}) \operatorname{SLP}(\theta, \phi),(\mathrm{h}) S(\theta, \phi),(\mathrm{i}) \operatorname{CRE}(\theta, \phi),(\mathrm{j})-\nabla \cdot \operatorname{AET}(\theta, \phi)$, and (k) cloud fraction at $(\theta, \phi)$, all regressed against $T(\theta, \phi)$. The spatially weighed global mean value is displayed above each panel. The zero isopleth is contoured black in each panel. All fluxes are positive into the atmospheric column. Stippling in (a)-(k) represents where over $90 \%$ of the AOGCMs agreed on the sign of the regression coefficient. (1)-(v) As in (a)-(k), but for observations. Note that (a) and (1) are the same as Figs. 1b and 1c respectively but are reproduced here for convenience.

(Webb and Lock 2013). The surface albedo component also plays an important role in the positive $\gamma_{N}(\theta, \phi)$ relationship over the Southern Ocean, near the Arctic sea ice margin, and over the high altitude Rockies and Himalayan mountain ranges (Figs. 3b,m) where warm years are associated with less snow or sea ice.

The $\gamma_{N}(\theta, \phi)$ relationship (Figs. 3a,i) tends to be negative near both poles and over some specific continental regions (e.g., equatorial South America, equatorial Africa, Australia, and northern Eurasia). In these areas, the $\gamma_{\text {Clear Sw }}(\theta, \phi)$ (Figs. 3b,m) and $\gamma_{\mathrm{CRE}}(\theta, \phi)$ (Figs. 3i,t) components of $\gamma_{N}(\theta, \phi)$ are near zero. Since atmospheric water vapor in these locations is limited compared to the tropical ocean, the Planck response [embedded within the $\gamma_{\text {ClearLW }}(\theta, \phi)$ component; Figs. 3e,p] is able to emerge as the dominant influence on $\gamma_{N}(\theta, \phi)$.
Figure 3 also maps the $\gamma_{S}(\theta, \phi)$ relationship (Figs. $3 \mathrm{~h}, \mathrm{~s})$, which tends to be positive over the equatorial ocean where natural variability in the thermocline heat budget can cause persistent, large-magnitude $T(\theta, \phi)$ anomalies (Deser et al. 2010). In this part of the globe, $\gamma_{S}(\theta, \phi)$ is much larger than $\gamma_{N}(\theta, \phi)$, indicating that it dominates the local energy budget. Furthermore, both the $\gamma_{S}(\theta, \phi)$ and the $\gamma_{N}(\theta, \phi)$ relationships are positive over much of the equatorial ocean (see Figs. 3a,h and 31,s), indicating that $T(\theta, \phi)$ anomalies in these location cannot be damped locally and tend to be associated with anomalous atmospheric energy transport, which communicates local anomalous $S(\theta, \phi)$ to higher latitudes (Kosaka and Xie 2013). The $\gamma_{-\nabla \cdot A E T}(\theta, \phi)$ relationship (Figs. 3j,u) shows that warm $T(\theta, \phi)$ anomalies over the equatorial ocean and portions of the subtropics are associated with 
Variables as a function of both climatological $T(\theta, \phi)$ and anomalous $T(\theta, \phi)$ over land

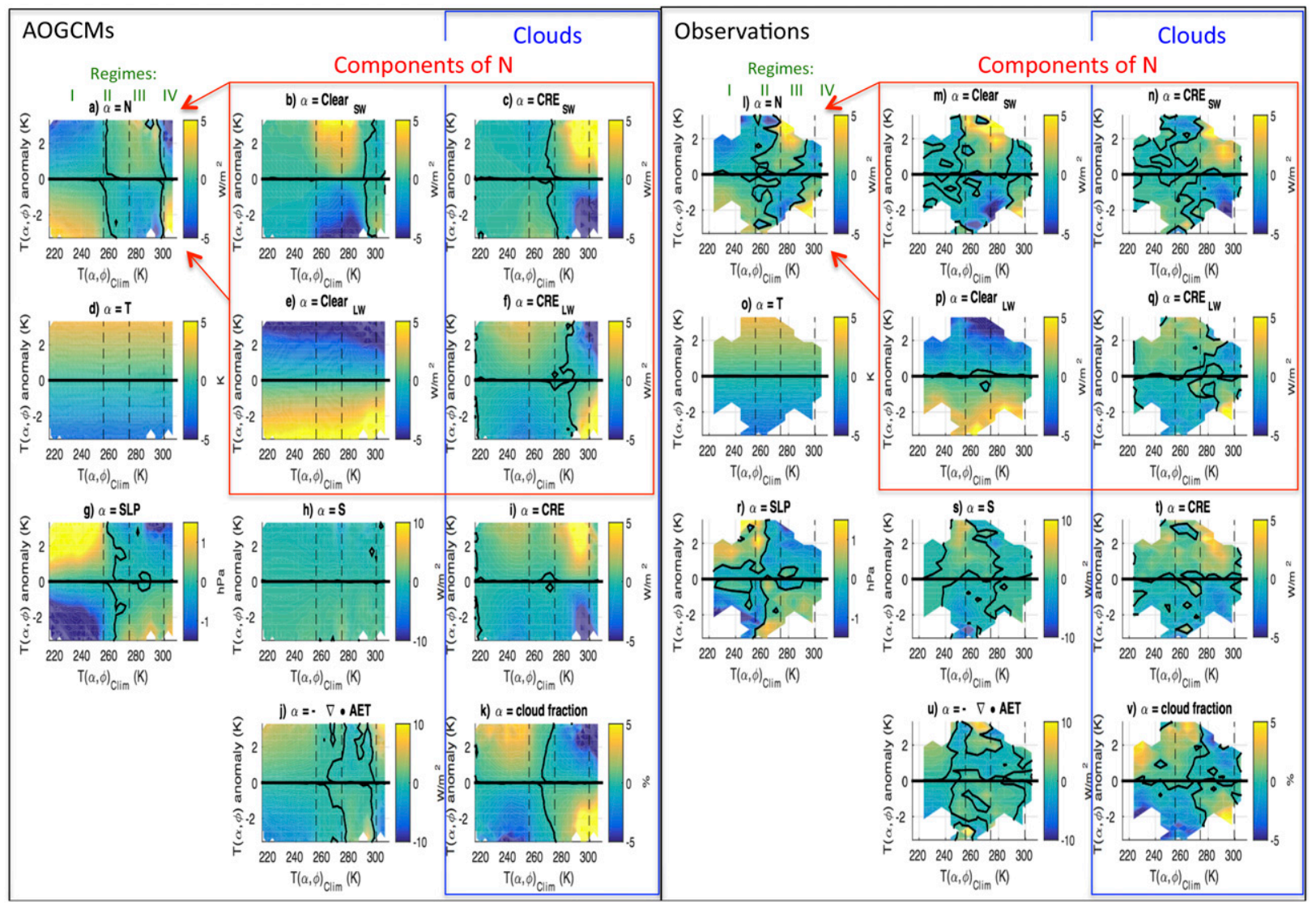

FIG. 4. Dependency of relationships on local climatological temperature, $T(\theta, \phi)_{\text {Clim. }}$ Bivariate plots over land grid points for the same variables from Fig. 3 plotted as a function of climatological $T(\theta, \phi)\left[T(\theta, \phi)_{\text {Clim }}\right]$ and $T(\theta, \phi)$ anomaly. Regimes discusses in the text are labeled in (a) and (l) and are delineated in the plots with vertical dashed lines. Figure S3 in the supplementary material contours the mean geographical extent of the regimes. These panels are produced by sampling every grid point and every year, pooling the data into two-dimensional histograms, and averaging over the pooled values to produce the number displayed (in the observations and AOGCMs separately). The bins and the number of data points underlying each average value are shown in Fig. S2. White areas had no values to calculate an average.

net anomalous horizontal export of energy while warm $T(\theta, \phi)$ anomalies over many continental and highlatitude regions are associated with the net anomalous horizontal import of energy from other locations.

\section{Dependency of the $\gamma_{N}(\theta, \phi)$ relationship on climatological $T(\theta, \phi)$}

The geographic distribution apparent in Fig. 3 suggests that the physics of the $\gamma_{N}(\theta, \phi)$ relationship may depend fundamentally on the climatological value of $T(\theta, \phi)\left[T(\theta, \phi)_{\text {Clim }}\right]$ as well as whether the location is over land or ocean. Figures 4 and 5 illustrate how the variables shown in Fig. 3 vary as a function of $T(\theta, \phi)_{\mathrm{Clim}}$ and anomalous $T(\theta, \phi)$ over land (Fig. 4) and ocean (Fig. 5) grid points. The bins and the number of data points underlying each average value are shown in Fig. S2 of the supplementary material. Figures 4a, 4l, $5 \mathrm{a}$, and 51 label four regimes [regime I with $T(\theta, \phi)_{\mathrm{Clim}}$ values below $255 \mathrm{~K}$; regime II with $T(\theta, \phi)_{\text {Clim }}$ values from 255 to $273 \mathrm{~K}$; regime III with $T(\theta, \phi)_{\text {Clim }}$ values from 273 to $300 \mathrm{~K}$; and regime IV with $T(\theta, \phi)_{\text {Clim }}$ values above $300 \mathrm{~K}$ ] that were chosen to highlight noteworthy shifts in the underlying physical mechanisms of the $N(\theta, \phi)$ versus $T(\theta, \phi)$ relationship.

All four regimes indicate that over land, elevated $T(\theta, \phi)$ anomalies are associated with a negative Clear $_{\text {LW }}(\theta, \phi)$ contribution to $N(\theta, \phi)$ (Figs. 4e,p) via the Planck response. Over the ocean, however, the strong water vapor feedback overwhelms the Planck response near $300 \mathrm{~K}$ in the AOGCMs (Fig. 5e), but this feature is not present in observations (Fig. 5p) as was discussed in section 3. Since there is no water vapor runaway greenhouse effect over land, the anomalous $N(\theta, \phi)$ 
Variables as a function of both climatological $T(\theta, \phi)$ and anomalous $T(\theta, \phi)$ over ocean

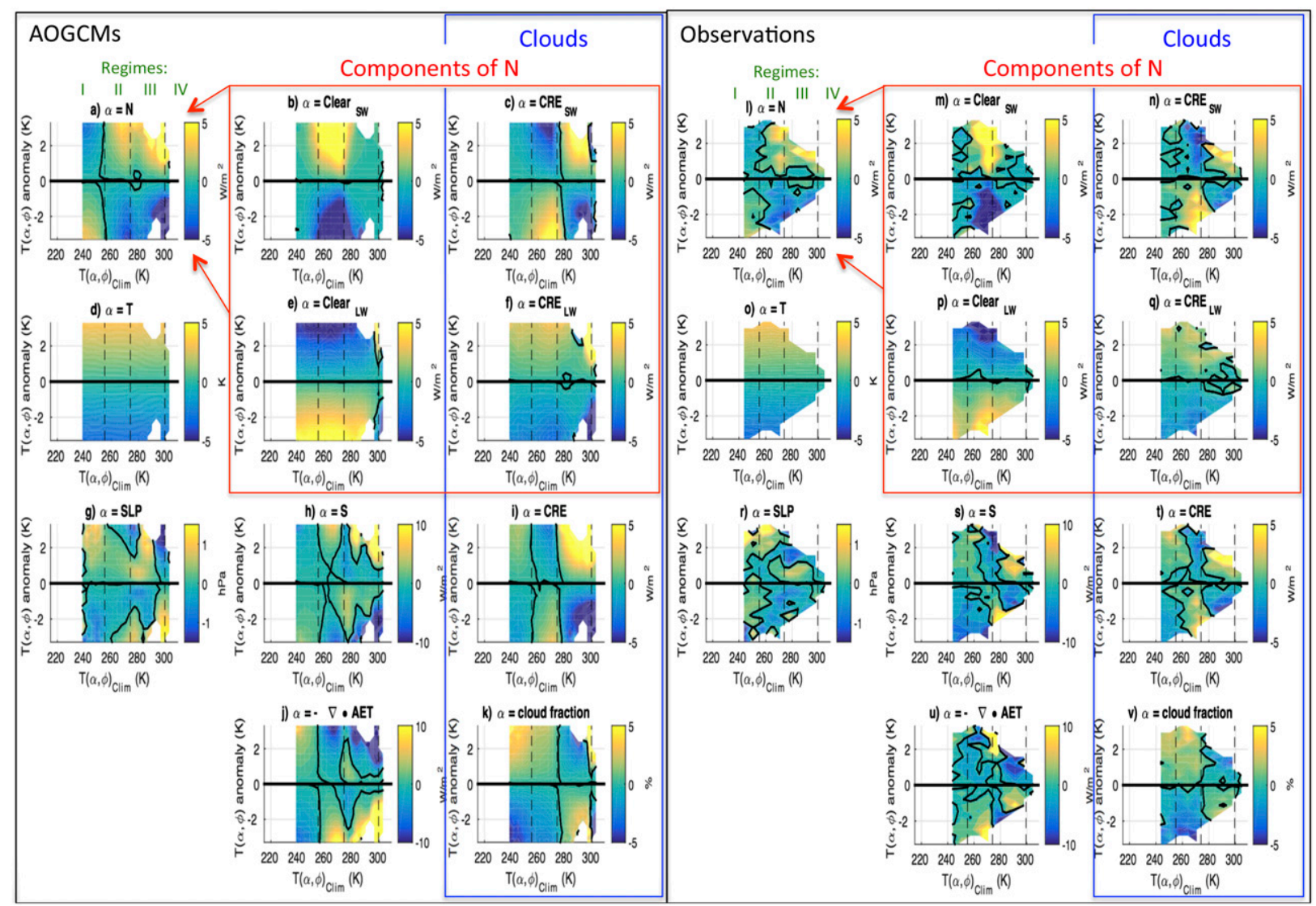

FIG. 5. As in Fig. 4, but over ocean grid points.

versus $T(\theta, \phi)$ relationship (Figs. 4a,l) is governed by the ability of the surface albedo (Figs. $4 \mathrm{~b}, \mathrm{~m})$ and $\operatorname{CRE}(\theta, \phi)$ components (Figs. 4i,t) to overwhelm the $\operatorname{Clear}_{\mathrm{LW}}(\theta, \phi)$ component.

Over regime I, cold climatological $T(\theta, \phi)$ values, which are well below the freezing point of water, produce semipermanent ice that is not prone to variation. Consequently, there is little shortwave variability in this regime over land or ocean from either $\operatorname{Clear}_{s w}(\theta, \phi)$ (Figs. 4b,m and 5b,m) or $\mathrm{CRE}_{\mathrm{Sw}}(\theta, \phi)$ (Figs. $4 \mathrm{c}, \mathrm{n}$ and $5 \mathrm{c}, \mathrm{n})$. Additionally, the shortwave components of variability make less of an impact in this regime because these locations are at high latitudes and experience less annually averaged incoming solar radiation than the rest of the planet. Anomalous warmth in regime I is associated with increased cloud fraction (Figs. $4 \mathrm{k}, \mathrm{v}$ and $5 \mathrm{k}, \mathrm{v}$ ) and a positive $\operatorname{CRE}_{\mathrm{LW}}(\theta, \phi)$ and $\operatorname{CRE}(\theta, \phi)$ anomaly (Figs. 4i,t and $5 \mathrm{i}, \mathrm{t}$ ); however, this effect is not large enough to overwhelm the $\operatorname{Clear}_{\mathrm{LW}}(\theta, \phi)$ response (Figs. 4e,p and 5e,p). This implies that anomalous warmth over Antarctica and the polar Arctic Ocean (likely caused by anomalous convergence of AET;
Figs. $4 \mathrm{j}, \mathrm{u}$ and $5 \mathrm{j}, \mathrm{u}$ ) will tend to be strongly damped by the Planck response.

Unlike regime I, regime II experiences anomalously positive $N(\theta, \phi)$ during positive $T(\theta, \phi)$ anomalies. Regime II is characterized by $T(\theta, \phi)_{\text {Clim }}$ values near the freezing point of water so positive $T(\theta, \phi)$ anomalies are associated with significant reductions in surface albedo over land and ocean (Figs. 4b,m and 5b,m). These reductions in surface albedo are larger than the negative $\operatorname{Clear}_{\mathrm{LW}}(\theta, \phi)$ response (Figs. $4 \mathrm{e}$ and 5e,p), except in observations over land where the $\operatorname{Clear}_{\mathrm{LW}}(\theta, \phi)$ mostly overwhelms the $\operatorname{Clear}_{\mathrm{sw}}(\theta, \phi)$ component (Fig. 4l) but this may be an artifact of a limited number of observations (Fig. S2b).

Regime III also tends to experience anomalously positive $N(\theta, \phi)$ during positive $T(\theta, \phi)$ anomalies. Regime III, is generally above the freezing point of water and thus it is the $\operatorname{CRE}_{\mathrm{SW}}(\theta, \phi)$ component that is primarily responsible (Figs. $4 \mathrm{c}, \mathrm{n}$ and $5 \mathrm{c}, \mathrm{n}$ ) for the positive $N(\theta, \phi)$ versus $T(\theta, \phi)$ relationship. In this regime, anomalous warmth is associated with a reduction in cloud fraction (Figs. $4 \mathrm{k}, \mathrm{v}$ and $5 \mathrm{k}, \mathrm{v}$ ) that causes a larger 
reduction in cloud albedo (Figs. $4 \mathrm{c}, \mathrm{n}$ and $5 \mathrm{c}, \mathrm{n}$ ) than cloud greenhouse effect (Figs. 4f,q and 5f,q). The direction of causality is particularly ambiguous in this regime since reduced cloudiness leads to warmth (Trenberth and Shea 2005).

Over land, where the water vapor supply is limited, $T(\theta, \phi)$ warmth in regime IV is associated with anomalously negative $N(\theta, \phi)$ (Figs. 4a,l). In this regime, anomalous warmth is associated with decreased precipitation (Trenberth and Shea 2005) and cloud fraction (Figs. 4k,v); however, because of longwave and shortwave cancellation, the $\operatorname{CRE}(\theta, \phi)$ response is relatively small (Figs. 4i,t). Also, since the $T(\theta, \phi)_{\text {clim }}$ value is well above the freezing point of water, the $\operatorname{Clear}_{\mathrm{Sw}}(\theta, \phi)$ response is near zero. These factors allow the Planck response (embedded in Figs. $4 \mathrm{e}$ and $4 \mathrm{p}$ ) to dominate the total response (Figs. 4a,1). Like regime I, regime IV over land tends to be an area of AET convergence during anomalous $T(\theta, \phi)$ warmth (Figs. $4 \mathrm{j}, \mathrm{u})$.

\section{The negative $\overline{\gamma_{N}}$ relationship}

Having established some of the underlying physics governing the geographic distribution of the local $N(\theta, \phi)$ versus $T(\theta, \phi)$ relationship, we now turn our attention to the problem of reconciling the mostly positive local $N(\theta, \phi)$ versus $T(\theta, \phi)$ relationship (Figs. 1b-d and 3a,1) with the negative $\bar{N}$ versus $\bar{T}\left(\overline{\gamma_{N}}\right)$ relationship (Fig. 1a). One possible way to square these seemingly paradoxical results would be through the specific spatial pattern of $T(\theta, \phi)$ anomalies associated with changes in $\bar{T}$ [i.e., $\zeta_{T}(\theta, \phi)$; Eq. (12)]. Specifically, we showed in sections 3 and 4 that certain locations on the surface of the planet are better able to damp $T(\theta, \phi)$ anomalies to space than others. For example, anomalous warmth over Antarctica and the polar Arctic Ocean will tend to be effectively damped by the Planck response (Figs. 1b-d and 3a,1). Therefore, if $\zeta_{T}(\theta, \phi)$ was distributed such that most of the anomalous warmth was in high-latitude regions characterized by a negative $\gamma_{N}(\theta, \phi)$ relationship, then the apparent contradiction of Fig. 1a and Figs. 1b-d might be resolved.

Figure 6 displays the $\zeta_{T}(\theta, \phi)$ pattern (Figs. 6d,o) as well as the corresponding, $\zeta_{\alpha}(\theta, \phi)$ for all the other variables shown in Figs. 3-5. On the interannual time scale, variability in $\bar{T}$ is dominated by $\mathrm{El} \mathrm{Niño-}$ Southern Oscillation (ENSO; Wigley 2000), which has a distinct $\zeta_{T}(\theta, \phi)$ pattern (Brown et al. 2014b). Importantly, the $\zeta_{T}(\theta, \phi)$ pattern does include large positive values at high latitudes where $\gamma_{N}(\theta, \phi)$ tends to be negative and the $\zeta_{T}(\theta, \phi)$ pattern includes negative values over some locations with a locally positive $\gamma_{N}(\theta$, $\phi)$ relationship (cf. Figs. 6d,o and 3a,1). The high-latitude amplification in the characteristic $\zeta_{T}(\theta, \phi)$ pattern is partly a result of a large surface energy flux $\left[\zeta_{s}(\theta, \phi)\right.$; Figs. 6h,s] from the tropical Pacific that is transferred to high latitudes by the atmosphere where horizontal convergence occurs (Figs. 6j,u). The negative $\zeta_{T}(\theta, \phi)$ values in the North Pacific (Figs. 6d,o) occur due to an atmospheric circulation response to enhanced convection at the equator during El Niño (Trenberth et al. 1998), which strengthens the Aleutian low (Figs. 6g,r). The deeper Aleutian low implies anomalous northerly (southerly) winds over the northwestern (northeastern) Pacific and thus anomalously negative (positive) $\zeta_{T}(\theta, \phi)$ (Alexander et al. 2002; Emery and Hamilton 1985; Lau and Nath 1994). There is also an anomalously cool region in the South Pacific off the coast of Australia that arises primarily due to a shift in the South Pacific convergence zone (SPCZ) during El Niño (Folland et al. 2002).

These surface temperature features play a role in the production of the negative $\bar{N}$ versus $\bar{T}$ relationship. In AOGCMs, $-0.4 \mathrm{~W} \mathrm{~m}^{-2} \mathrm{~K}^{-1}$ of the $-0.8 \mathrm{~W} \mathrm{~m}^{-2} \mathrm{~K}^{-1}$ $\overline{\zeta_{N}(\theta, \phi)}$ originates from locations with that are warm when the global mean is warm and have a negative local feedback [i.e., positive $\zeta_{T}(\theta, \phi)$ and negative $\gamma_{N}(\theta, \phi)$ ] like Antarctica (Fig. 7). Also, $-0.5 \mathrm{~W} \mathrm{~m}^{-2} \mathrm{~K}^{-1}$ of the $-0.8 \mathrm{~W} \mathrm{~m}^{-2} \mathrm{~K}^{-1} \overline{\zeta_{N}(\theta, \phi)}$ originates from locations that are cool when the global mean is warm but have a positive local feedback [i.e., negative $\zeta_{T}(\theta, \phi)$ and positive $\left.\gamma_{N}(\theta, \phi)\right]$ like the northwestern Pacific (Fig. 7). Similarly, in observations $-1.2 \mathrm{~W} \mathrm{~m}^{-2} \mathrm{~K}^{-1}$ of the $-2.4 \mathrm{~W} \mathrm{~m}^{-2} \mathrm{~K}^{-1} \overline{\zeta_{N}(\theta, \phi)}$ originates from locations with positive $\zeta_{T}(\theta, \phi)$ and negative $\gamma_{N}(\theta, \phi)$ while $-1.3 \mathrm{~W} \mathrm{~m}^{-2} \mathrm{~K}^{-1}$ originates from locations with negative $\zeta_{T}(\theta, \phi)$ and positive $\gamma_{N}(\theta, \phi)$ (Fig. 7). This is consistent with the finding that AOGCMs with more Arctic amplification in their subdecadal $\zeta_{T}(\theta, \phi)$ pattern have less variable $\bar{T}$ (Brown et al. 2014b) due to more of the $\bar{T}$ weighting being in regions where energy can be easily damped to space. However, the $\zeta_{N}(\theta, \phi)$ spatial pattern (Figs. 6a,1) has other unique characteristics (Dessler 2013; Koumoutsaris 2013; Trenberth et al. 2010) that are not explained by the superposition of $\zeta_{T}(\theta, \phi)$ (Figs. 6d,o) and $\gamma_{N}(\theta, \phi)$ (Figs. 3a,1).

To quantify the component of $\zeta_{N}(\theta, \phi)$ that is not explained by the surface temperature pattern associated with $\bar{T}$ variability, we follow a procedure similar to Armour et al. (2013) where we multiply the local feedback relationships $\gamma_{\alpha}(\theta, \phi)$ (Fig. 3) by the characteristic surface temperature pattern associated with $\bar{T}$ variability, $\zeta_{T}(\theta, \phi)$ (Figs. 6d,o):

$$
\kappa_{\alpha}(\theta, \phi)=\gamma_{\alpha}(\theta, \phi) \zeta_{T}(\theta, \phi) .
$$




$$
\zeta_{\alpha}(\theta, \emptyset)=\frac{\Delta[\alpha(\theta, \emptyset)]}{\Delta \bar{T}} \text { (where } \alpha=\text { any variable) } \quad \text { (i.e. what happens when global } \mathrm{T} \text { is high...) }
$$

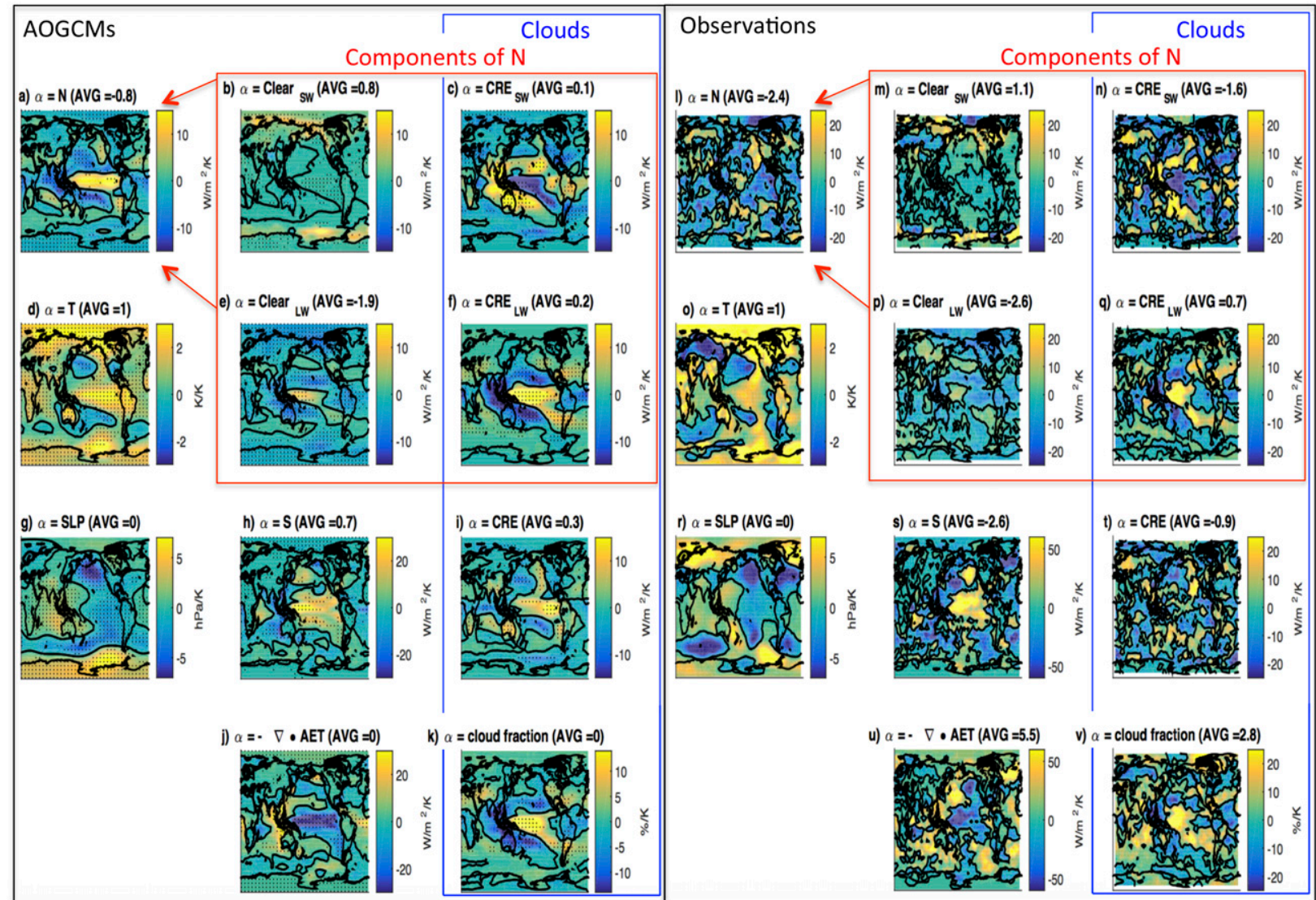

FIG. 6. As in Fig. 3, but local variables are regressed against global mean temperature, $\bar{T}$ [Eq. (12)], rather than local temperature, $T(\theta, \phi)$. Note that the magnitude of the response for observations is larger than that for AOGCMs and that is partially because differences in the location of sharp gradients in the AOGCMs will result in smaller absolute values when the average is taken. [The global mean of each map is the $L=0$ value in Fig. 9 and the global mean of (a) and (1) is represented in Fig. 1a.]

This calculation, shown in Fig. 8, illustrates what $\zeta_{\alpha}(\theta, \phi)$ would be if local $T(\theta, \phi)$ explained $100 \%$ of the variance in local $\alpha(\theta, \phi)$. For AOGCMs the $\kappa_{N}(\theta, \phi)$ pattern is qualitatively similar to the $\zeta_{N}(\theta, \phi)$ pattern (cf. Figs. 6a and $8 \mathrm{a})$. In particular, both $\zeta_{N}(\theta, \phi)$ and $\kappa_{N}(\theta, \phi)$ have strong positive values over the tropical Pacific and more negative values over the anomalously cool subtropical Pacific, continents, and high latitudes. However, the surface temperature pattern by itself significantly underpredicts the $\uparrow \mathrm{LW}$ damping of $\bar{T}$ anomalies. In particular, $\overline{\kappa_{\text {ClearLw }}(\theta, \phi)}=-1.1 \mathrm{~W} \mathrm{~m}^{-2}$ (Fig. 8e) is less negative than $\overline{\zeta_{\text {Clear LW }}(\theta, \phi)}=-1.9 \mathrm{~W} \mathrm{~m}^{-2}$ (Fig. 6e), and $\overline{\kappa_{\text {CRELW }}(\theta, \phi)}=1.7 \mathrm{~W} \mathrm{~m}^{-2}$ (Fig. 8f) is far more positive than $\overline{\zeta_{\text {CRELW }}(\theta, \phi)}=0.2 \mathrm{~W} \mathrm{~m}^{-2}$ (Fig. 6f). For observations, there is less qualitative similarity between the $\kappa_{N}(\theta, \phi)$ and $\zeta_{N}(\theta, \phi)$ patterns (cf. Figs. 61 and 81). It is not surprising that the patterns in the observations contain less coherent structure than the corresponding patterns in the AOGCMs given that the observed regression coefficients are based on 14 years while the AOGCM regression coefficients are based on 5400 years (200 years each for 27 AOGCMs). Nevertheless, observations also show that the surface temperature pattern times the local feedback underestimates the $\uparrow \mathrm{LW}$ damping of $\bar{T}$ anomalies. This results from $\overline{\kappa_{\text {Clear LW }}(\theta, \phi)}=-1.5 \mathrm{~W} \mathrm{~m}^{-2}$ (Fig. $8 \mathrm{p}$ ) being less negative than $\overline{\zeta_{\text {Clear LW }}(\theta, \phi)}=-2.6 \mathrm{~W} \mathrm{~m}^{-2}$ (Fig. $6 \mathrm{p}$ ).

For both AOGCMs and observations, $\overline{\kappa_{N}(\theta, \phi)}$ is positive [1.1 $\mathrm{W} \mathrm{m}^{-2}$ (Fig. 8a) and $0.9 \mathrm{~W} \mathrm{~m}^{-2}$ (Fig. 81)], indicating that the superposition of the characteristic surface temperature pattern associated with $\bar{T}$ variability and the local feedback would, by itself, produce a positive $\overline{\gamma_{N}}$ and would be indicative of an unstable climate system. This implies that the mechanisms other than the characteristic surface temperature pattern associated with $\bar{T}$ variability must be crucial for stabilizing $\bar{T}$ to internal perturbations. To highlight the contribution from these other mechanisms we subtract $\kappa_{\alpha}(\theta, \phi)$ 


\begin{tabular}{c|c|c|c|c|}
\cline { 2 - 5 } & \multicolumn{2}{|c|}{ AOGCMs (total = -0.8) } & \multicolumn{2}{c|}{ Observations (total = -2.4) } \\
\hline & $\zeta_{T}(\theta, \emptyset)>0$ & $\zeta_{T}(\theta, \varnothing)<0$ & $\zeta_{T}(\theta, \varnothing)>0$ & $\zeta_{T}(\theta, \varnothing)<0$ \\
\hline$\gamma_{N}(\theta, \varnothing)>0$ & 0.2 & -0.5 & 0.0 & -1.3 \\
\hline$\gamma_{N}(\theta, \varnothing)<0$ & -0.4 & 0.0 & -1.2 & 0.1 \\
\hline
\end{tabular}

FIG. 7. Values of $\zeta_{N}(\theta, \phi)$ [Eq. (12); Figs. 6a,1] averaged over locations with the indicated properties of $\zeta_{T}(\theta, \phi)$ [Eq. (12); Figs. $6 \mathrm{~d}, \mathrm{o}]$ and $\gamma_{N}(\theta, \phi)$ [Eq. (11); Figs. 3a,1]. Units for all values are $\mathrm{W} \mathrm{m}{ }^{-2} \mathrm{~K}^{-1}$. Colors give an indication of relative magnitude with reds representing positive values and blues representing negative values.

(Fig. 8) from the directly simulated or observed relationship $\zeta_{\alpha}(\theta, \phi)$ (Fig. 6),

$$
\omega_{\alpha}(\theta, \phi)=\zeta_{\alpha}(\theta, \phi)-\kappa_{\alpha}(\theta, \phi),
$$

and plot these values in Fig. 9.

This calculation reveals that $\kappa_{\alpha}(\theta, \phi)$ greatly underpredicts the magnitude of negative $N$ values over much of the surface of the planet, particularly over the Pacific tropics and subtropics (Figs. 9a,1). It is well known that positive $\bar{T}$ (and thus positive ENSO) is associated with a great amount of heat flux from the Pacific Ocean to the atmosphere (Trenberth et al. 2002a; see Figs. 6h,s herein). This anomalous heat flux causes a large reorganization of the atmospheric circulation that leads to a strengthening of the Hadley cell over the Pacific and alters the Walker circulation leading to anomalous subsidence over Indonesia (Klein et al. 1999). Figure 9 indicates that these ENSO-specific atmospheric features are not heavily tied to the characteristic $T(\theta, \phi)$ pattern. In particular, the patterns of $\zeta_{S}(\theta, \phi)$ (Figs. 6h,s) and $\zeta_{S L P}(\theta, \phi)$ (Figs. 6g,r) are very similar to their corresponding patterns of $\omega_{S}(\theta, \phi)$ (Figs. 9h,s) and $\omega_{\text {SLP }}(\theta, \phi)$ (Figs. 9g,r).

These ENSO-caused shifts in $S$ and large-scale atmospheric circulation have a profound impact on the $\omega_{N}(\theta, \phi)$ pattern (Figs. 9a,l). In particular, the large negative $\omega_{N}(\theta, \phi)$ values over Indonesia and the equatorial Atlantic are associated with anomalously high $\omega_{\mathrm{SLP}}(\theta, \phi)$ (Figs. 9g,r), indicating that these are regions of anomalous subsidence during positive $\bar{T}$ that are not caused by the local $T(\theta, \phi)$ $\kappa_{\alpha}(\theta, \emptyset)=\mu_{\alpha}(\theta, \varnothing) \cdot \zeta_{T}(\theta, \varnothing) \quad($ where $\alpha=$ any variable $)$

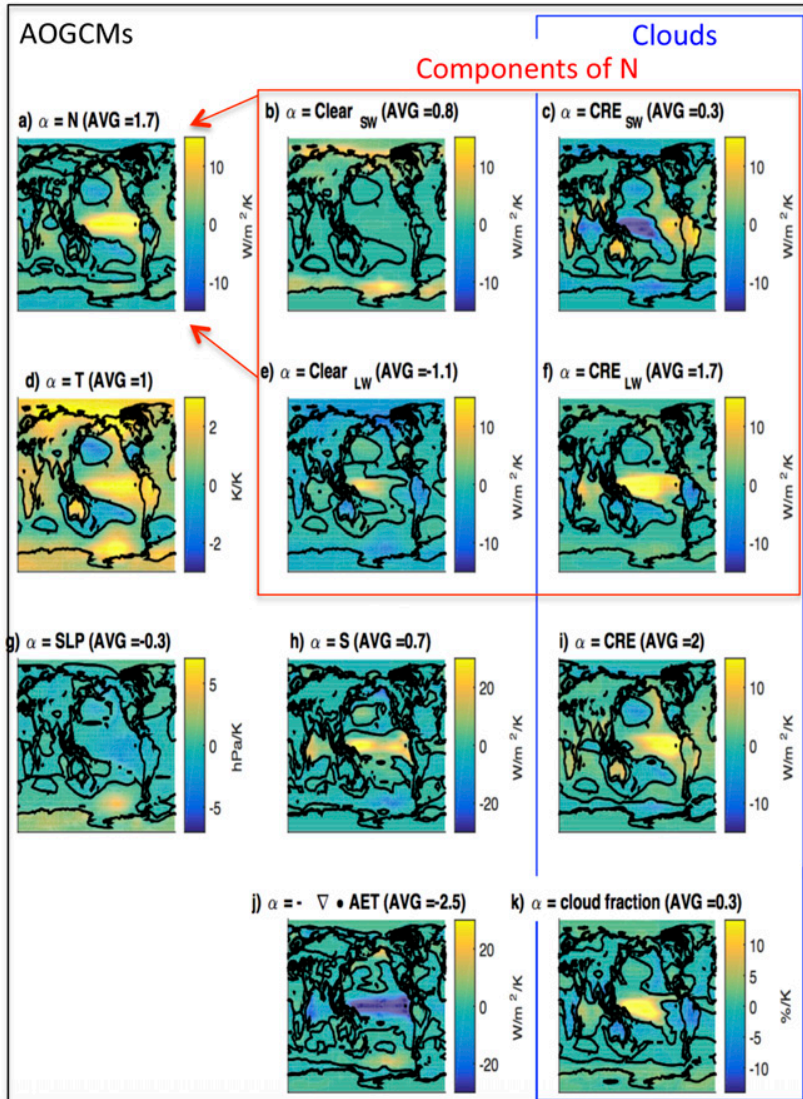

(i.e. what happens when global $\mathrm{T}$ is high because of local feedbacks and local T...)

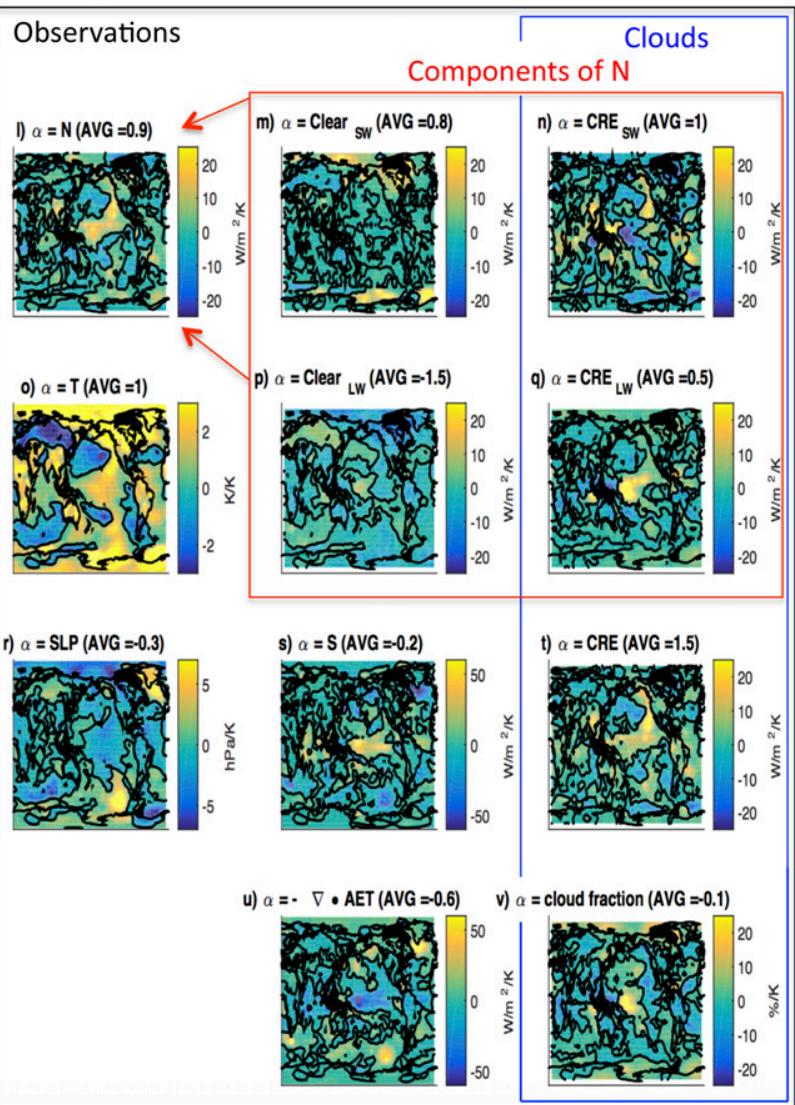

FIG. 8. The portion of the $\zeta_{\alpha}(\theta, \phi)$ pattern (Fig. 6) due to the characteristic surface temperature pattern associated with $\bar{T}$ variability $\left[\zeta_{T}(\theta, \phi)\right.$; Figs. $\left.6 \mathrm{~d}, \mathrm{o}\right]$ multiplied by the local feedback pattern $\left[\gamma_{\alpha}(\theta, \phi) ;\right.$ Fig. 3]; see Eq. (13). 


$$
\omega_{\alpha}(\theta, \varnothing)=\zeta_{\alpha}(\theta, \varnothing)-\kappa_{\alpha}(\theta, \varnothing) \quad(\text { where } \alpha=\text { any variable })
$$

(i.e. what happens when global $\mathrm{T}$ is high but not because of local feedbacks and local T...)

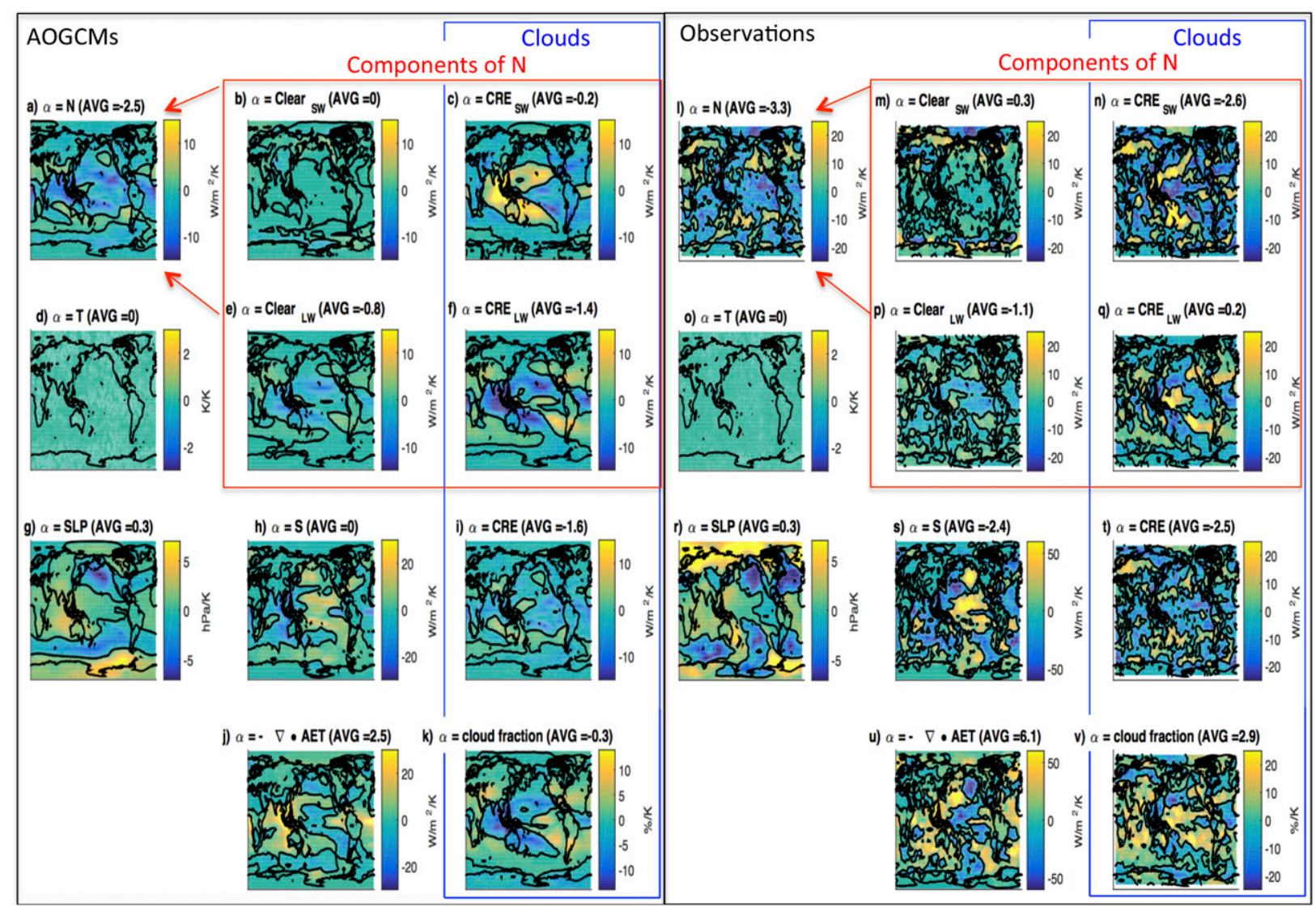

FIG. 9. The portion of the $\zeta_{\alpha}(\theta, \phi)$ pattern (Fig. 6) that is not due to the characteristic surface temperature pattern associated with $\bar{T}$ variability $\left[\zeta_{T}(\theta, \phi)\right.$; Figs. 6d,o] multiplied by the local feedback pattern $\left[\gamma_{\alpha}(\theta, \phi)\right.$; Fig. 3]; see Eq. (14).

anomaly. This subsidence is associated with reduced cloud fraction (Figs. 9k,v) and a negative $\omega_{\mathrm{CRE}}(\theta, \phi)$ response (Figs. 9i,t), due mostly to a negative $\omega_{\text {CRE LW }}(\theta, \phi)$ response (Figs. 9f,q). The negative $\omega_{\mathrm{CRELW}}(\theta, \phi)$ values in these regions may also be influenced by reduced cloud height (Allan et al. 2002; Cess et al. 2001). Additionally, there is a circulation-induced negative $\omega_{\mathrm{Clear} L W}(\theta, \phi)$ response in these regions (Figs. 9e,p) associated with drying of the of the middle to upper troposphere during ENSO events (Colman and Power 2010; Dessler 2013; Koumoutsaris 2013; Nilsson and Emanuel 1999; Pierrehumbert 1995). The decoupling of TOA net radiation from the surface temperature pattern in this region is consistent with previous findings showing that $\uparrow \mathrm{LW}$ in the tropics is controlled much more by middle and upper tropospheric water vapor than by local surface temperature (Allan et al. 1999).

The analysis above demonstrates that at the peak of an unforced $\bar{T}$ anomaly, the negative $\bar{N}$ versus $\bar{T}$ relationship results largely from mechanisms other than the spatial distribution of the characteristic surface temperature pattern associated with the $\bar{T}$ anomaly (Figs. 9a,l). However, the above analysis has not indicated when, over the course of an unforced $\bar{T}$ anomaly, the decoupling between the actual TOA radiation response (i.e., $\overline{\gamma_{N}}$ ) and that expected from the characteristic surface temperature pattern [i.e., $\overline{\kappa_{N}(\theta, \phi)}$ ] takes place. We investigate this question by calculating regressions of global mean variables, $\bar{\alpha}$, against $\bar{T}$ at time lags of $L$ years,

$$
\left[\overline{\gamma(L)_{\alpha}}\right]=\left\{\frac{\Delta[\bar{\alpha}(L)]}{\Delta[\bar{T}(L=0)]}\right\}
$$

and comparing these with the corresponding values that would be expected if the local feedback, $\gamma_{\alpha}(\theta, \phi)$, and the characteristic surface temperature pattern, $\zeta_{T}(\theta, \phi)$, explained $100 \%$ of the variability:

$\left[\overline{\kappa(L)_{\alpha}}\right]=\frac{1}{A} \sum_{i}^{M} a_{i}\left[\gamma_{\alpha}(\theta, \phi, L=0) \zeta_{T}(\theta, \phi, L)\right]_{i}$ 

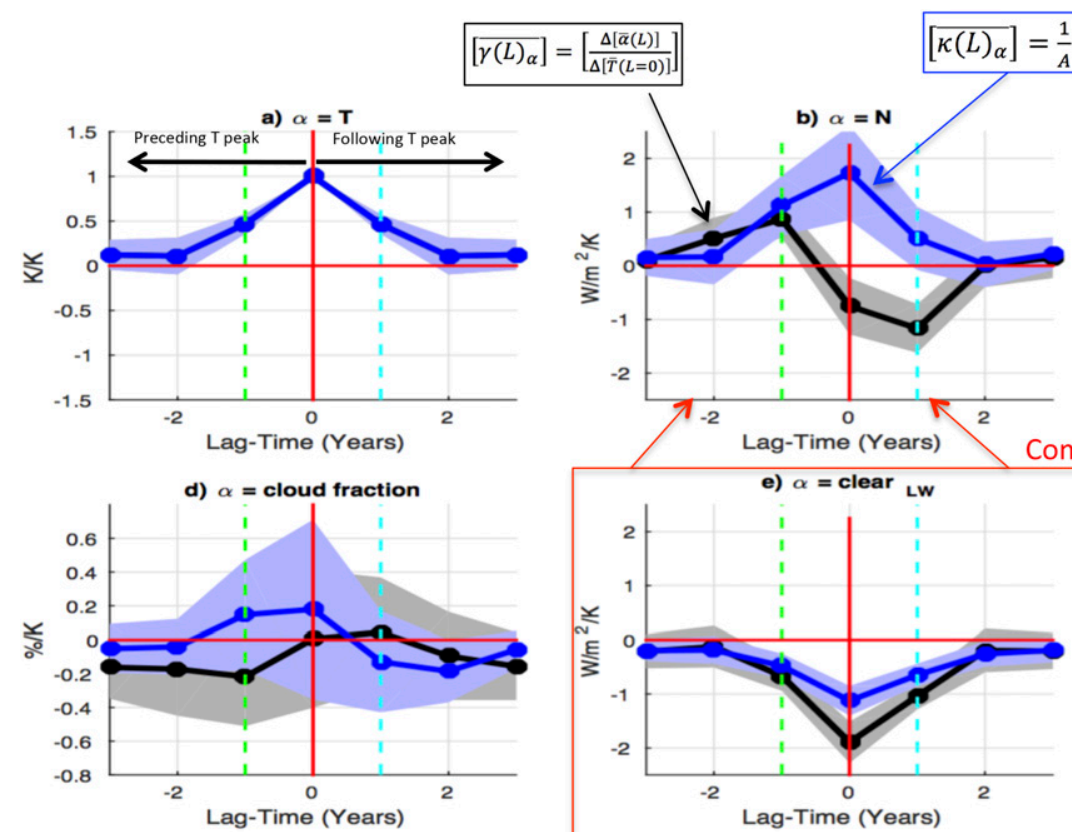

$\left[\kappa(L)_{\alpha}\right]=\frac{1}{A} \sum_{i}^{M} a_{i} \cdot\left[\gamma_{\alpha}(\theta, \varnothing, L=0) \cdot \zeta_{T}(\theta, \emptyset, L)\right]_{i}$
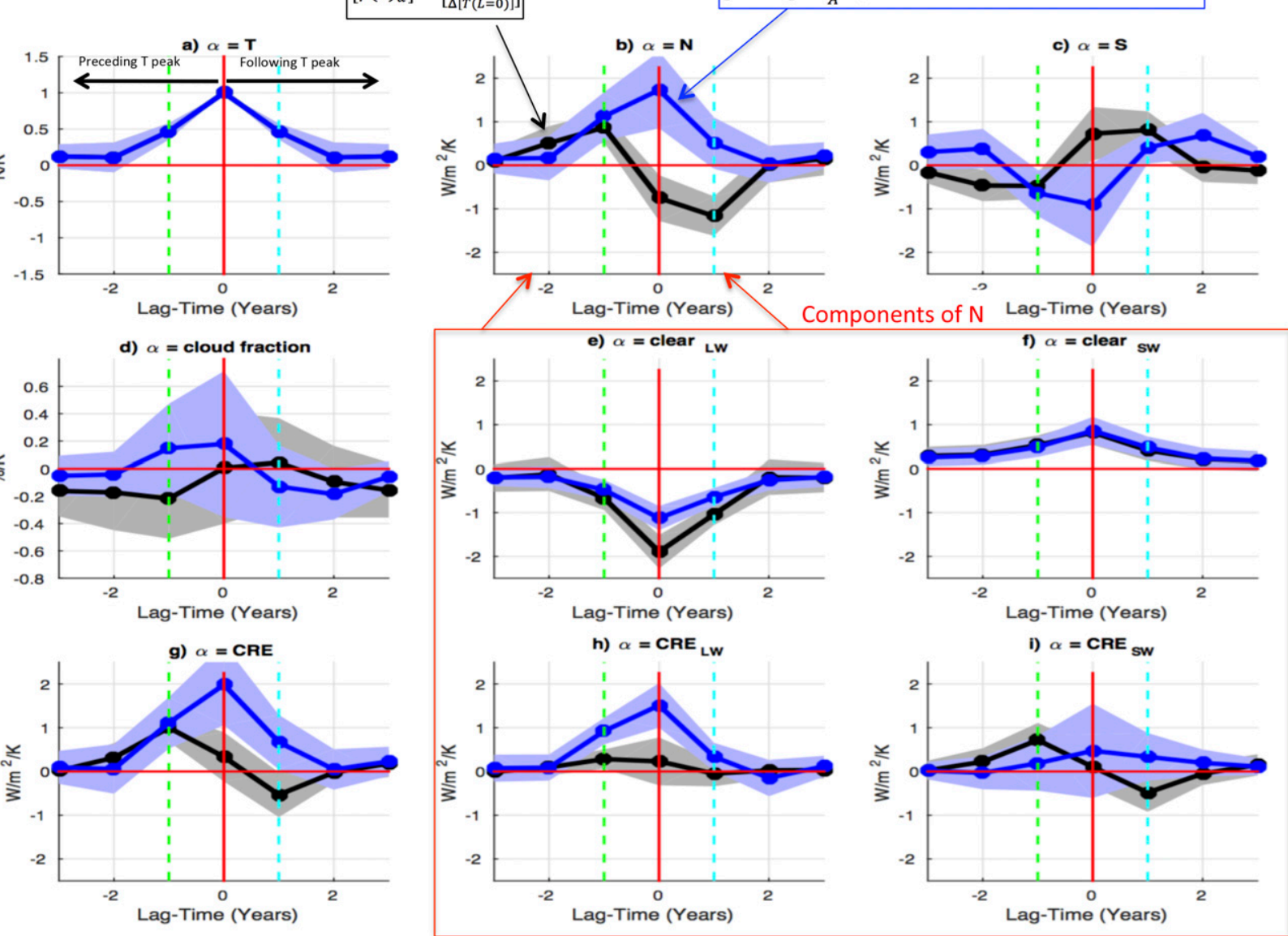

FIG. 10. Cross regression between global mean values and $\bar{T}\left[\overline{\gamma(L)_{\alpha}}\right]$, as well as the cross regression that would be expected if the $\zeta_{T}(\theta, \phi)$ pattern and the local $\gamma_{\alpha}(\theta, \phi)$ feedback explained $100 \%$ of the variability $\left[\overline{\kappa(L)_{\alpha}}\right]$; see Eq. (16). This analysis was conducted for AOGCMs only. Shading represents the across-AOGCM standard deviation of the regression values.

The comparison of Eqs. (15) and (16) is shown in Fig. 10. Many years are required to perform robust crossregressions so we omit the observational data from this portion of the analysis. Figure 10 shows that from 3 years to 1 year prior to a typical $\bar{T}$ maximum, a positive net TOA energy imbalance $\left[\overline{\gamma(L)_{N}}\right]$ develops, reaching a maximum of $0.87 \mathrm{~W} \mathrm{~m}^{-2} \mathrm{~K}^{-1}$ the year before the $\bar{T}$ maximum (Fig. 10b). The development of this positive $\overline{\gamma(L)_{N}}$ anomaly is largely due to the characteristic surface temperature pattern $\left[\zeta_{T}(\theta, \phi, L)\right]$ itself since $\overline{\kappa(L)_{N}}$ nearly matches $\overline{\gamma(L)_{N}}$ from $L=-3$ to $L=-1$ (Fig. 10b). The positive net TOA energy imbalance $\left[\overline{\gamma(L)_{N}}\right]$ is associated with a negative net surface heat flux imbalance $\left[\overline{\gamma(L)_{S}}\right]$ at $L=-1$, indicating that the ocean tends to be absorbing net energy from space in the year leading up to the maximum in $\bar{T}$ (Fig. 10c).

The positive progression of the net TOA energy imbalance $\left[\overline{\gamma(L)_{N}}\right]$ from $L=-3$ to $L=-1$ is largely due to the cloud radiative effect component $\left[\overline{\gamma(L)_{\mathrm{CRE}}}\right]$, which ascends in accord with expectations based on the characteristic surface temperature pattern $\left[\zeta_{T}(\theta, \phi, L)\right.$; Fig. $10 \mathrm{~g}$. Since ENSO variability leads $\bar{T}$ variability in time (Trenberth et al. 2002a), it is likely that the positive $\overline{\gamma(L)_{\mathrm{CRESW}}}$ and $\overline{\gamma(L)_{\mathrm{CRELW}}}$ at $L=-1$ represent positive cloud feedbacks operating in conjunction with local ENSO activity in the Pacific (Radley et al. 2014).

Between $L=-1$ and $L=0$, the net surface heat flux imbalance $\left[\overline{\gamma(L)_{S}}\right]$ switches sign from negative to positive, indicating a large release of energy from the ocean to the atmosphere (Trenberth et al. 2014). It is over this period that the $\zeta_{T}(\theta, \phi, L)$ pattern predicts an increase in the net TOA energy imbalance $\left[\overline{\gamma(L)_{N}}\right.$; i.e., an increase in $\left.\overline{\kappa(L)_{N}}\right]$ but AOGCMs actually simulate a drop in $\overline{\gamma(L)_{N}}$ from positive to negative (Figs. 10b, 6a, and 1a). The $\zeta_{T}(\theta, \phi, L)$ pattern nearly perfectly predicts the $\overline{\gamma(L)_{\text {Clearsw }}}$ ice albedo component (Figs. 10f and 9b) but it underestimates the magnitude of the negative $\overline{\gamma(L)_{\text {Clear LW }}}$ component (Figs. 10e and 9e) and greatly overestimates the positive 
$\overline{\gamma(L)_{\text {CRE LW }}}$ component. The implication is that during the peak of a positive $\bar{T}$ anomaly (i.e., an El Niño event), there is a great amount of heat flux from the ocean to the atmosphere where it can more easily be emitted to space in the form of $\uparrow \mathrm{LW}$. Additionally ENSO dynamics cause a large-scale rearrangement of atmospheric circulation that causes more efficient $\uparrow$ LW (Figs. 10e,h) due to drying and reduced cloud fraction over large portions of Indo-Pacific tropics and subtropics. Overall, this causes the net TOA energy imbalance $\left[\overline{\gamma(L)_{N}}\right]$ to reduce to a negative value at $L=0$ despite $\overline{\kappa(L)_{N}}$ continuing in its positive ascent (Fig. 10b).

A year after the maximum in $\bar{T}, \overline{\gamma(L)_{\text {Clear Sw }}}$ remains

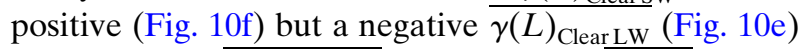
and a negative $\overline{\gamma(L)_{\text {CRE LW }}}$ (Fig. 10h) help $\overline{\gamma(L)_{N}}$ remain negative despite the tendency of the characteristic surface temperature pattern $\left[\zeta_{T}(\theta, \phi, L)\right]$ to induce a positive TOA net radiation imbalance $\left[\overline{\kappa(L)_{N}}\right.$; Fig. 10b]. This negative TOA net radiation imbalance acts as a restoring force, causing the $\bar{T}$ anomaly to return to its equilibrium value.

\section{Summary}

In order for the unforced climate system to be stable in the long run, it is expected that the global mean TOA net radiation imbalance, $\bar{N}$, will exhibit a negative relationship with unforced global mean surface temperature anomalies, $\bar{T}$. We show that this negative relationship exists in both contemporary observations as well as in state-of-the-art AOGCMs. However, we also show that, at the local spatial scale, the simultaneous relationship between $N(\theta, \phi)$ and $T(\theta, \phi)$ tends to be positive over most of the surface of the planet. The reasons for the positive relationship differ by geographic location and have a strong dependence on the climatological mean $T(\theta, \phi)$. The locally positive relationship is mostly due to the surface shortwave component (i.e., ice albedo feedback) for regions with $T(\theta, \phi)_{\text {clim }}$ values near the freezing point of water, mostly due to the shortwave cloud radiative effect component over regions with intermediate to high $T(\theta, \phi)_{\text {clim }}$ values (from $\sim 273$ to $\sim 300 \mathrm{~K}$ ), and mostly due to the longwave water vapor feedback over oceanic regions with the highest $T(\theta, \phi)_{\text {clim values. }}$

The mostly positive $N(\theta, \phi)$ versus $T(\theta, \phi)$ relationship at the local spatial scale can be reconciled with the globally negative $\bar{N}$ versus $\bar{T}$ relationship when anomalous atmospheric energy transport, the characteristic surface temperature pattern, and adjustments in the large-scale atmospheric circulation are considered. In particular, positive $\bar{T}$ anomalies are associated with El Niño events in which there is large anomalous heat flux from the Pacific Ocean into the atmosphere where the local $N(\theta, \phi)$ versus $T(\theta, \phi)$ relationship is positive. This leads to significant horizontal divergence of atmospheric energy transport over the tropical $\mathrm{Pa}$ cific, and convergence of atmospheric energy transport at high latitudes and specific continental regions. This redistribution of energy helps create a characteristic $T(\theta, \phi)$ versus $\bar{T}$ pattern with a substantial amount of warmth at high latitudes [characterized by a locally negative $N(\theta, \phi)$ versus $T(\theta, \phi)$ relationship where the temperature anomaly can be more easily damped to space]. Additionally, the characteristic $T(\theta, \phi)$ versus $\bar{T}$ pattern contains anomalously $\operatorname{cool} T(\theta, \phi)$ regions where a locally positive $N(\theta, \phi)$ versus $T(\theta, \phi)$ relationship promotes a locally negative $N(\theta, \phi)$.

However, the characteristic $T(\theta, \phi)$ versus $\bar{T}$ pattern by itself cannot explain the negative $\bar{N}$ versus $\bar{T}$ relationship because a multiplication of the local $N(\theta, \phi)$ versus $T(\theta, \phi)$ map by the $T(\theta, \phi)$ versus $\bar{T}$ map produces a positive estimate of the $\bar{N}$ versus $\bar{T}$ relationship. This indicates that atmospheric circulation changes associated with unforced interannual $\bar{T}$ variability are crucial in the explanation of the negative $\bar{N}$ versus $\bar{T}$ relationship. In particular, a $\bar{T}$ maximum is preceded, a year prior, by a positive $\bar{N}$ that is consistent with expectations based on the $T(\theta, \phi)$ versus $\bar{T}$ pattern. However, simultaneous to the $\bar{T}$ peak, a great rearrangement of large-scale atmospheric circulation causes reduced cloud cover and subsidence-induced drying in broad regions of the tropical and subtropical Indo-Pacific. This circulation change allows for much more efficient release of $\uparrow \mathrm{LW}$ energy than would otherwise be expected from the $T(\theta, \phi)$ versus $\bar{T}$ pattern alone. Because the short time scale relationship between $\bar{N}$ and $\bar{T}$ is heavily influenced by large-scale atmospheric circulation changes (opposed to local feedbacks), this study supports the notion that there may be very little relationship between the climate feedback parameter (i.e., $\gamma_{N}$ ) diagnosed from annual or subannual time scale variability and $2 \times \mathrm{CO}_{2}$ equilibrium climate sensitivity.

Acknowledgments. We thank Dr. Drew Shindell for helpful discussions on this topic. We acknowledge Dr. Aaron Donohoe and two anonymous reviewers whose comments greatly enhanced the manuscript. We acknowledge the World Climate Research Programme's Working Group on Coupled Modelling, which is responsible for CMIP, and we thank the climate modeling groups for producing and making available their model output. For CMIP the U.S. Department of Energy's Program for Climate Model Diagnosis and 
Intercomparison provides coordinating support and led development of software infrastructure in partnership with the Global Organization for Earth System Science Portals. This work was partially supported by NSF Grant AGS-1147608. We also acknowledge the support from NASA ROSES13-NDOA, ROSES12-MAP, and ROSES-NEWS programs. This research was partially conducted at the Jet Propulsion Laboratory, California Institute of Technology, sponsored by NASA.

\section{REFERENCES}

Alexander, M. A., I. Bladé, M. Newman, J. R. Lanzante, N.-C. Lau, and J. D. Scott, 2002: The atmospheric bridge: The influence of ENSO teleconnections on air-sea interaction over the global oceans. J. Climate, 15, 2205-2231, doi:10.1175/ 1520-0442(2002)015<2205:TABTIO > 2.0.CO;2.

Allan, R. P., K. P. Shine, A. Slingo, and J. A. Pamment, 1999: The dependence of clear-sky outgoing long-wave radiation on surface temperature and relative humidity. Quart. J. Roy. Meteor. Soc., 125, 2103-2126, doi:10.1002/ qj. 49712555809 .

__- A. Slingo, and M. A. Ringer, 2002: Influence of dynamics on the changes in tropical cloud radiative forcing during the 1999 El Niño. J. Climate, 15, 1979-1986, doi:10.1175/ 1520-0442(2002)015<1979:IODOTC>2.0.CO;2.

—, C. Liu, N. G. Loeb, M. D. Palmer, M. Roberts, D. Smith, and P.-L. Vidale, 2014: Changes in global net radiative imbalance 1985-2012. Geophys. Res. Lett., 41, 5588-5597, doi:10.1002/ 2014 GL060962.

Armour, K. C., C. M. Bitz, and G. H. Roe, 2013: Time-varying climate sensitivity from regional feedbacks. J. Climate, 26, 4518-4534, doi:10.1175/JCLI-D-12-00544.1.

Bellenger, H., E. Guilyardi, J. Leloup, M. Lengaigne, and J. Vialard, 2014: ENSO representation in climate models: From CMIP3 to CMIP5. Climate Dyn., 42, 1999-2018, doi:10.1007/s00382-013-1783-z.

Bellomo, K., A. C. Clement, T. Mauritsen, G. Rädel, and B. Stevens, 2014: Simulating the role of subtropical stratocumulus clouds in driving Pacific climate variability. J. Climate, 27, 5119-5131, doi:10.1175/JCLI-D-13-00548.1.

,,$-- \ldots$, and -2015 : The influence of cloud feedbacks on equatorial Atlantic variability. J. Climate, 28, 2725-2744, doi:10.1175/JCLI-D-14-00495.1.

Bony, S., and Coauthors, 2006: How well do we understand and evaluate climate change feedback processes? J. Climate, 19, 3445-3482, doi:10.1175/JCLI3819.1.

Brown, P. T., W. Li, L. Li, and Y. Ming, 2014a: Top-of-atmosphere radiative contribution to unforced decadal global temperature variability in climate models. Geophys. Res. Lett., 41, 51755183, doi:10.1002/2014GL060625.

,-- , and S.-P. Xie, 2014b: Regions of significant influence on unforced global mean surface air temperature variability in climate models. J. Geophys. Res. Atmos., 120, 480-494, doi:10.1002/2014JD022576.

,,-- E. C. Cordero, and S. A. Mauget, 2015: Comparing the model-simulated global warming signal to observations using empirical estimates of unforced noise. Sci. Rep., 5, 9957, doi:10.1038/srep09957.

Cess, R. D., M. Zhang, B. A. Wielicki, D. F. Young, X.-L. Zhou, and Y. Nikitenko, 2001: The influence of the 1998
El Niño upon cloud-radiative forcing over the Pacific warm pool. J. Climate, 14, 2129-2137, doi:10.1175/ 1520-0442(2001)014<2129:TIOTEN >2.0.CO;2.

Chen, X., and K.-K. Tung, 2014: Varying planetary heat sink led to global-warming slowdown and acceleration. Science, $\mathbf{3 4 5}$, 897-903, doi:10.1126/science.1254937.

Colman, R., and S. Power, 2010: Atmospheric radiative feedbacks associated with transient climate change and climate variability. Climate Dyn., 34, 919-933, doi:10.1007/ s00382-009-0541-8.

Crook, J. A., P. M. Forster, and N. Stuber, 2011: Spatial patterns of modeled climate feedback and contributions to temperature response and polar amplification. J. Climate, 24, 3575-3592, doi:10.1175/2011JCLI3863.1.

Dee, D. P., and Coauthors, 2011: The ERA-Interim reanalysis: Configuration and performance of the data assimilation system. Quart. J. Roy. Meteor. Soc., 137, 553-597, doi:10.1002/qj.828.

Deser, C., M. A. Alexander, S.-P. Xie, and A. S. Phillips, 2010: Sea surface temperature variability: Patterns and mechanisms. Annu. Rev. Mar. Sci., 2, 115-143, doi:10.1146/ annurev-marine-120408-151453.

Dessler, A. E., 2010: A determination of the cloud feedback from climate variations over the past decade. Science, 330, 15231527, doi:10.1126/science. 1192546.

2013: Observations of climate feedbacks over 2000-10 and comparisons to climate models. J. Climate, 26, 333-342, doi:10.1175/JCLI-D-11-00640.1.

Dolinar, E., X. Dong, B. Xi, J. Jiang, and H. Su, 2015: Evaluation of CMIP5 simulated clouds and TOA radiation budgets using NASA satellite observations. Climate Dyn., 44, 2229-2247, doi:10.1007/s00382-014-2158-9.

Donohoe, A., and D. S. Battisti, 2011: Atmospheric and surface contributions to planetary albedo. J. Climate, 24, 4402-4418, doi:10.1175/2011JCLI3946.1.

Drijfhout, S. S., A. T. Blaker, S. A. Josey, A. J. G. Nurser, B. Sinha, and M. A. Balmaseda, 2014: Surface warming hiatus caused by increased heat uptake across multiple ocean basins. Geophys. Res. Lett., 41, 7868-7874, doi:10.1002/2014GL061456.

Emery, W. J., and K. Hamilton, 1985: Atmospheric forcing of interannual variability in the northeast Pacific Ocean: Connections with El Niño. J. Geophys. Res., 90, 857-868, doi:10.1029/ JC090iC01p00857.

England, M. H., and Coauthors, 2014: Recent intensification of wind-driven circulation in the Pacific and the ongoing warming hiatus. Nat. Climate Change, 4, 222-227, doi:10.1038/ nclimate2106.

Evan, A. T., R. J. Allen, R. Bennartz, and D. J. Vimont, 2013: The modification of sea surface temperature anomaly linear damping time scales by stratocumulus clouds. J. Climate, 26, 3619-3630, doi:10.1175/JCLI-D-12-00370.1.

Folland, C. K., J. A. Renwick, M. J. Salinger, and A. B. Mullan, 2002: Relative influences of the interdecadal Pacific oscillation and ENSO on the South Pacific convergence zone. Geophys. Res. Lett., 29, 1643, doi:10.1029/2001GL014201.

Hallberg, R., and A. K. Inamdar, 1993: Observations of seasonal variations in atmospheric greenhouse trapping and its enhancement at high sea surface temperature. J. Climate, 6, 920-931, doi:10.1175/1520-0442(1993)006<0920:OOSVIA > 2.0.CO;2.

Hasselmann, K., 1976: Stochastic climate models. Part I: Theory. Tellus, 28A, 473-485, doi:10.1111/j.2153-3490.1976.tb00696.x.

Hawkins, E., and R. Sutton, 2009: The potential to narrow uncertainty in regional climate predictions. Bull. Amer. Meteor. Soc., 90, 1095-1107, doi:10.1175/2009BAMS2607.1. 
Inamdar, A. K., and V. Ramanathan, 1994: Physics of greenhouse effect and convection in warm oceans. J. Climate, 7, 715-731, doi:10.1175/1520-0442(1994)007<0715:POGEAC >2.0.CO;2.

Ingram, W., 2013: Some implications of a new approach to the water vapour feedback. Climate Dyn., 40, 925-933, doi:10.1007/ s00382-012-1456-3.

Kato, S., 2009: Interannual variability of the global radiation budget. J. Climate, 22, 4893-4907, doi:10.1175/2009JCLI2795.1.

Kiehl, J. T., 1994: On the observed near cancellation between longwave and shortwave cloud forcing in tropical regions. J. Climate, 7, 559-565, doi:10.1175/1520-0442(1994)007<0559: OTONCB $>2.0 . \mathrm{CO} ; 2$.

Klein, S. A., B. J. Soden, and N.-C. Lau, 1999: Remote sea surface temperature variations during ENSO: Evidence for a tropical atmospheric bridge. J. Climate, 12, 917-932, doi:10.1175/ 1520-0442(1999)012<0917:RSSTVD>2.0.CO;2.

Kosaka, Y., and S.-P. Xie, 2013: Recent global-warming hiatus tied to equatorial Pacific surface cooling. Nature, 501, 403-407, doi:10.1038/nature12534.

Koumoutsaris, S., 2013: What can we learn about climate feedbacks from short-term climate variations? Tellus, $\mathbf{6 5 \mathbf { A }}, 18887$, doi:10.3402/tellusa.v65i0.18887.

Larson, K., and D. L. Hartmann, 2003: Interactions among cloud, water vapor, radiation, and large-scale circulation in the tropical climate. Part I: Sensitivity to uniform sea surface temperature changes. J. Climate, 16, 1425-1440, doi:10.1175/ 1520-0442-16.10.1425.

Lau, N.-C., and M. J. Nath, 1994: A modeling study of the relative roles of tropical and extratropical SST anomalies in the variability of the global atmosphere-ocean system. J. Climate, 7, 1184-1207, doi:10.1175/1520-0442(1994)007<1184: AMSOTR $>2.0 . \mathrm{CO} ; 2$.

Loeb, N., and Coauthors, 2012: Advances in understanding top-ofatmosphere radiation variability from satellite observations. Surv. Geophys., 33, 359-385, doi:10.1007/s10712-012-9175-1.

Meehl, G. A., A. Hu, J. M. Arblaster, J. Fasullo, and K. E. Trenberth, 2013: Externally forced and internally generated decadal climate variability associated with the interdecadal Pacific oscillation. J. Climate, 26, 7298-7310, doi:10.1175/ JCLI-D-12-00548.1.

Minnis, P., and Coauthors, 2011: CERES edition-2 cloud property retrievals using TRMM VIRS and Terra and Aqua MODIS data-Part I: Algorithms. IEEE Trans. Geosci. Remote Sens., 49, 4374-4400.

Nilsson, J., and K. A. Emanuel, 1999: Equilibrium atmospheres of a two-column radiative-convective model. Quart. J. Roy. Meteor. Soc., 125, 2239-2264, doi:10.1002/qj.49712555814.

Palmer, M. D., and D. J. McNeall, 2014: Internal variability of Earth's energy budget simulated by CMIP5 climate models. Environ. Res. Lett., 9, 034016, doi:10.1088/1748-9326/9/3/ 034016.

Park, S., C. Deser, and M. A. Alexander, 2005: Estimation of the surface heat flux response to sea surface temperature anomalies over the global oceans. J. Climate, 18, 4582-4599, doi:10.1175/JCLI3521.1.

Pierrehumbert, R. T., 1995: Thermostats, radiator fins, and the local runaway greenhouse. J. Atmos. Sci., 52, 1784-1806, doi:10.1175/1520-0469(1995)052<1784:TRFATL >2.0.CO;2.

Radley, C., S. Fueglistaler, and L. Donner, 2014: Cloud and radiative balance changes in response to ENSO in observations and models. J. Climate, 27, 3100-3113, doi:10.1175/JCLI-D-13-00338.1.
Ramanathan, V., and W. Collins, 1991: Thermodynamic regulation of ocean warming by cirrus clouds deduced from observations of the 1987 El Niño. Nature, 351, 27-32, doi:10.1038/351027a0.

- , R. D. Cess, E. F. Harrison, P. Minnis, B. R. Barkstrom, E. Ahmad, and D. Hartmann, 1989: Cloud-radiative forcing and climate: Results from the Earth Radiation Budget Experiment. Science, 243, 57-63, doi:10.1126/science.243.4887.57.

Smith, D. M., and Coauthors, 2015: Earth's energy imbalance since 1960 in observations and CMIP5 models. Geophys. Res. Lett., 42, 1205-1213, doi:10.1002/2014GL062669.

Soden, B. J., A. J. Broccoli, and R. S. Hemler, 2004: On the use of cloud forcing to estimate cloud feedback. J. Climate, 17, 3661-3665, doi:10.1175/1520-0442(2004)017<3661:OTUOCF $>2.0 . C O ; 2$.

Su, H., W. G. Read, J. H. Jiang, J. W. Waters, D. L. Wu, and E. J. Fetzer, 2006: Enhanced positive water vapor feedback associated with tropical deep convection: New evidence from Aura MLS. Geophys. Res. Lett., 33, L05709, doi:10.1029/ 2005 GL025505.

Taylor, K. E., R. J. Stouffer, and G. A. Meehl, 2012: An overview of CMIP5 and the experiment design. Bull. Amer. Meteor. Soc., 93, 485-498, doi:10.1175/BAMS-D-11-00094.1.

Trenberth, K. E., and D. J. Shea, 2005: Relationships between precipitation and surface temperature. Geophys. Res. Lett., 32, L14703, doi:10.1029/2005GL022760.

— , G. W. Branstator, D. Karoly, A. Kumar, N.-C. Lau, and C. Ropelewski, 1998: Progress during TOGA in understanding and modeling global teleconnections associated with tropical sea surface temperatures. J. Geophys. Res., 103, 14 291-14 324, doi:10.1029/97JC01444.

_ J. M. Caron, D. P. Stepaniak, and S. Worley, 2002a: Evolution of El Niño-Southern Oscillation and global atmospheric surface temperatures. J. Geophys. Res., 107, 4065, doi:10.1029/ 2000JD000298.

_ D. P. Stepaniak, and J. M. Caron, 2002b: Interannual variations in the atmospheric heat budget. J. Geophys. Res., 107, 4066, doi:10.1029/2000JD000297.

_ J. J. T. Fasullo, C. O’Dell, and T. Wong, 2010: Relationships between tropical sea surface temperature and top-of-atmosphere radiation. Geophys. Res. Lett., 37, L03702, doi:10.1029/ 2009GL042314.

,-- , and M. A. Balmaseda, 2014: Earth's energy imbalance. J. Climate, 27, 3129-3144, doi:10.1175/JCLI-D-13-00294.1.

_ , Y. Zhang, J. T. Fasullo, and S. Taguchi, 2015: Climate variability and relationships between top-of-atmosphere radiation and temperatures on Earth. J. Geophys. Res. Atmos., 120, 3642-3659, doi:10.1002/2014JD022887.

Trzaska, S., A. W. Robertson, J. D. Farrara, and C. R. Mechoso, 2007: South Atlantic variability arising from air-sea coupling: Local mechanisms and tropical-subtropical interactions. J. Climate, 20, 3345-3365, doi:10.1175/JCLI4114.1.

Webb, M. J., and A. Lock, 2013: Coupling between subtropical cloud feedback and the local hydrological cycle in a climate model. Climate Dyn., 41, 1923-1939, doi:10.1007/s00382-012-1608-5.

Wielicki, B. A., B. R. Barkstrom, E. F. Harrison, R. B. Lee, G. Louis Smith, and J. E. Cooper, 1996: Clouds and the Earth's Radiant Energy System (CERES): An Earth observing system experiment. Bull. Amer. Meteor. Soc., 77, 853-868, doi:10.1175/1520-0477(1996)077<0853:CATERE >2.0.CO;2.

Wigley, T. M. L., 2000: ENSO, volcanoes and record-breaking temperatures. Geophys. Res. Lett., 27, 4101-4104, doi:10.1029/ 2000 GL012159. 